\title{
Fixed Point Theory and the Liouville-Caputo Integro-Differential FBVP with Multiple Nonlinear Terms
}

\author{
Shahram Rezapour $\mathbb{D}^{1,},{ }^{1,2}$ Ali Boulfoul, ${ }^{3}$ Brahim Tellab ${ }^{D},{ }^{3}$ Mohammad Esmael Samei $\mathbb{D}^{4}$, \\ Sina Etemad $(\mathbb{D}), 1$ and Reny George ${ }^{10}{ }^{5,6}$ \\ ${ }^{1}$ Department of Mathematics, Azarbaijan Shahid Madani University, Tabriz, Iran \\ ${ }^{2}$ Department of Medical Research, China Medical University Hospital, China Medical University, Taichung, Taiwan \\ ${ }^{3}$ Laboratory of Applied Mathematics, Kasdi Merbah University, Ouargla B.P. 511 30000, Algeria \\ ${ }^{4}$ Department of Mathematics, Faculty of Basic Science, Bu-Ali Sina University, Hamedan, Iran \\ ${ }^{5}$ Department of Mathematics, College of Science and Humanities in Al-Kharj, Prince Sattam Bin Abdulaziz University, \\ Al-Kharj 11942, Saudi Arabia \\ ${ }^{6}$ Department of Mathematics and Computer Science, St. Thomas College, Bhilai, Chhattisgarth 49006, India
}

Correspondence should be addressed to Reny George; renygeorge02@yahoo.com

Received 19 October 2021; Revised 6 January 2022; Accepted 17 January 2022; Published 24 February 2022

Academic Editor: Alexander Meskhi

Copyright (c) 2022 Shahram Rezapour et al. This is an open access article distributed under the Creative Commons Attribution License, which permits unrestricted use, distribution, and reproduction in any medium, provided the original work is properly cited.

\begin{abstract}
This work is reserved for the study of a special category of boundary value problems (BVPs) consisting of Liouville-Caputo integro-differential equations with multiple nonlinear terms. This fractional model and its boundary value conditions (BVCs) involve different simple $\mathbb{B V P}$ s, in which the second BVC as a linear combination of two Caputo derivatives of the unknown function equals a nonzero constant. The Banach principle gives a unique solution for this Liouville-Caputo $\mathbb{B} \mathbb{P}$. Further, the Krasnoselskii and Leray-Schauder criteria give the existence property regarding solutions of the mentioned problem. For each theorem, we provide an example based on the required hypotheses and derive numerical data in the framework of tables and figures to show the consistency of results from different points of view.
\end{abstract}

\section{Introduction}

In recent years, fractional differential equations have attracted the attention of many authors because of the numerous applications in various branches of science and engineering, in particular, fluid mechanics, image and signal processing, electromagnetic theory, potential theory, fractals theory, biology, control theory, viscoelasticity, and so on [1-3]. From the mathematical point of view, a number of researchers working on fractional calculus conduct their research in the field of applications of different fractional operators and various structures of $\mathbb{B V P S}$ in modeling abstract and real-world phenomena, but the discussion related to the fractional derivatives is an old problem and continues to receive many kinds of feedback. The physical aspect of the fractional derivative is now proved in many investigations. As we know, fractional-order derivatives have many advantages in comparison to the first-order derivatives. For example, one of the most simple examples in which the fractional derivative has a significant impact can be observed in diffusion processes. It is established that the subdiffusion is obtained when the order of the fractional derivative belongs to the interval $(0,1)$. Another impact of fractional derivatives can be observed in stability analysis. There are many differential equations that are not stable with the first-order derivative but are stable when we replace the first-order derivative by the fractional-order derivatives. By considering these cases, we can understand the importance of fractional operators, and the Liouville-Caputo derivative is one of the most important examples in this field. For better and more accurate 
simulations and better numerical results, we use the Liouville-Caputo derivative in this paper.

Along with these important abilities, fixed point theory is regarded as one of the most important tools to derive existence criterion of solutions. To better understand the subject, some research in this field can be enumerated. In [4], Ahmad and Agarwal turned to the existence of solution for several new structures of fractional $\mathbb{B} M P$ s via slit-strip BVCs. Then, Ahmad and Ntouyas [5] and Alsaedi et al. [6] investigated similar results regarding solutions of a sequential $\mathbb{B M P}$ s consisting of nonlocal integro-differential inclusions of the Caputo type. In [7], Boucenna et al. defined a nonlinear $\mathbb{P}$ on the Sobolev space and utilized the special operators for proving theorems with the help of some tools in functional analysis. Similarly, Azzaoui et al. [8] defined a Sobolev space again and derived the existence criterion for positive solutions on such a space. Bai and Sun [9] not only established the aforesaid existence criterion regarding positive solutions but also derived their multiplicity to a singular $\mathbb{B M P}$. In [10], Islam et al. proved some results about the existence of a solution of an infinite system of integral equations by using a new family of contractions entitled the generalized $\alpha$-admissible Hardy-Rogers contractions in cone $b_{2}$-metric spaces over Banach algebras. In [11], Shoaib et al. studied other existence results via $f$-contractions of Nadler type in 2020. After that, recently, Ali et al. [12] considered a nonlinear fractional differential equation equipped with the integral type boundary conditions and proved the existence results with the help of topological degree theory.

Recently, Boulfoul et al. [13] considered a weighted space of the Banach type by defining a nonlinear integro-differential $\mathbb{B M P}$ on an unbounded domain and checked two properties of existence and uniqueness under fixed point techniques. In the sequel of this path, a new type of generalized fractional operator in the Hilfer settings was utilized by Shatanawi et al. to prove the main existence conditions for a nonlocal multipoint $\mathbb{B M P}[14]$. Zada et al. [15] continued similar area of existence theory by studying an impulsive integro-differential $\mathbb{B V}$ in the sense of Riemann-Liouville and reviewed the property of the stability. In 2021, the authors in [16, 17] used two numerical algorithms for approximating solutions of two similar multiorder multiterm $\mathbb{B V P}$, with $\mathrm{RL}$ operators and the generalized RL- $\psi$-operators. By developing studies in this regard, new classes of $\mathbb{B M P}$ s were designed in the context of p-Laplacian operators. Khan et al. introduced an advanced singular fractional in the framework of the Atangana-Baleanu derivation operators along with $p$-Laplacian structure [18]; then, in another work, Hasib Khan et al. [19] extended the above system in the form of a $p$-Laplacian hybrid $\mathbb{B} M P$.

Thereafter, some researchers expanded their existence results on real systems and models. For example, Rizwan et al. [20] designed a switched system of coupled impulsive implicit model of Langevin equation, and both stability and existence theories can be found in their paper for such a fractional physical structure. Etemad et al. [21] continued their study by considering an inclusion $\mathbb{B V P}$ of the Caputo-Hadamard type and accomplished some results by terms of a new notion called end-points along with approximate property for these points. In the same year, Samei et al. [22] reformulated similar Caputo-Hadamard inclusion $\mathbb{B M} \mathbb{P}$ of the hybrid type and turned to deriving existence criteria. The theory of topological degree is another tool for obtaining some results regarding solutions of a multiterm delay $\mathbb{B M P}$ which Sher et al. implemented it in their newly published article [23]. Abdeljawad et al. [24] modeled a new fractional $\mathbb{B V P}$ and proved the relevant existence theorems on the extended b-metric space. Also, Boutiara et al. [25] applied the Caputo type and Erdélyi-Kober type operators for modeling a nonlocal fractional $\mathbb{B M} \mathbb{P}$ and deriving existence aspects of solutions.

Along with above works, some researchers generalized existence theorems by terms of the existing notions in quantum fractional calculus. For instance, Etemad et al. [26] investigated a 3 -point quantum inclusion $\mathbb{B} \mathbb{P}$ in the context of $\alpha$ - $\psi$-contractions. Sitthiwirattham [27] studied $q$-integro-difference $\mathbb{B V} \mathbb{P}$ containing different values of $q$ and orders, and Sitho et al. [28] designed a noninstantaneous impulsive $q$-integro-difference $\mathbb{B} \mathbb{P}$ with quantum Hahn operators. Samei et al. also introduced a singular quantum $\mathbb{B V} \mathbb{P}$ for the first time [29]. Even, some applications of fixed point can be followed in the papers regarding mathematical biological models (see [30-32]).

In [33], Ntouyas and Tariboon discussed the multiorder $\mathbb{B Q P}$ with a linear combination of fractional integrals in the BVCs:

$$
\left\{\begin{array}{l}
r \mathbb{D}_{0}^{\sigma_{1}} z(\mathfrak{g})+(1-\dot{r}) \mathbb{D}_{0}^{\sigma_{2}} z(\mathfrak{G})=\mathfrak{u}(\mathfrak{G}, z(\mathfrak{g})), \quad 0<\mathfrak{G}<\tau^{*}, \\
z(0)=0, \quad r \dot{r} \mathbb{D} I_{0}^{\hat{\gamma}_{1}} z\left(\tau^{*}\right)+(1-\dot{r}) \mathbb{D} I_{0}^{\hat{\gamma}_{2}} z\left(\tau^{*}\right)=a_{0},
\end{array}\right.
$$

where $\mathbb{D}_{0}^{\dot{\eta}}$ stands for the Riemann-Liouville $\dot{\eta}^{\text {th }}$ derivative with $\dot{\eta} \in\left\{\hat{\sigma}_{1}, \hat{\sigma}_{2}\right\}$ provided that $1<\hat{\sigma}_{1}, \hat{\sigma}_{2}<2$ and $\square_{0}^{\dot{\eta}}$ is the Riemann-Liouville $\dot{\eta}^{\text {th }}$ integral with $\dot{\eta} \in\left\{\hat{v}_{1}, \dot{v}_{2}\right\}, a_{0} \in \mathbb{R}$, $0<\dot{r} \leq 1$, and $0 \leq r \leq 1$. Green's function for this corresponding problem has been investigated and some existence results have been obtained using fixed point theorems. $\mathrm{Xu}$ et al. [34] turned to investigating the existence property and Hyers-Ulam stability to fractional multiple order $\mathbb{B} M \mathbb{P}$ :

$$
\left\{\begin{array}{l}
r \mathbb{D}_{0}^{\sigma} z(\mathfrak{g})+\mathbb{D}_{0}^{v^{\prime}} z(\mathfrak{g})=\mathfrak{u}(\mathfrak{g}, z(\mathfrak{g})), \quad 0<\mathfrak{g}<\tau^{*}, \\
z(0)=0, \quad \dot{r} \mathbb{D}_{0}^{\sigma_{1}} z\left(\tau^{*}\right)+\mathbb{\sigma}_{0}^{\sigma_{2}} z\left(\mathfrak{I}_{0}\right)=a_{0},
\end{array}\right.
$$

where $\mathbb{D}_{0}^{\sigma}$ and $\mathbb{D}_{0}^{v}$ are Riemann-Liouville fractional derivatives, with $1<\dot{\sigma} \leq 2$ and $1 \leq \dot{v}<\dot{\sigma}, 0<\dot{r} \leq 1,0 \leq \dot{r} \leq 1$, $0 \leq \dot{\sigma}_{1} \leq \dot{\sigma}-\dot{v}, \hat{\sigma}_{2} \geq 0, a_{0} \in \mathbb{R}$, and $0<\mathfrak{g}_{0}<\tau^{*}$.

Inspired by the works cited above and to continue the study of existence theory in the context of fractional $\mathbb{B M P s}$, we focus on surveying some results regarding solutions of the following Liouville-Caputo integro-differential $\mathbb{B} V \mathbb{P}$ : 


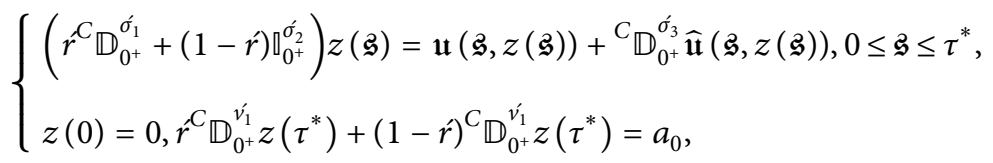

so that ${ }^{C} \mathbb{D}_{0^{+}}^{\dot{\eta}_{1}}$ is the Caputo $\dot{\eta}^{\text {th }}$ derivative with $\dot{\eta} \in\left\{\hat{\sigma}_{1}, \hat{\sigma}_{3}, \dot{v}_{1}, \dot{v}_{2}\right\}, a_{0} \in \mathbb{R}$ and $\mathbb{0}_{0^{+}}^{\sigma_{2}^{\prime}}$ stands for the Riemann-Liouville fractional $\hat{\sigma}_{2}^{\text {th }}$ integral such that $s 1<\hat{\sigma}_{1}, \hat{\sigma}_{3} \leq 2, \hat{\sigma}_{1}>\hat{\sigma}_{3}, \quad 0<\hat{\sigma}_{2} \leq 1, \quad 0<\dot{r} \leq 1, \quad 0 \leq \dot{r} \leq 1$, $0<\hat{v}_{1}, \hat{v}_{2}<\hat{\sigma}_{1}-\hat{\sigma}_{3}$, and $\mathfrak{u}, \widehat{\mathfrak{u}} \in C(\mathcal{O} \times \mathbb{R}, \mathbb{R})$ are two given functions, where $\mathscr{O}=:\left[0, \tau^{*}\right]$.

In fact, the existing ideas of two papers published in $[33,34]$ motivate us to design a combined model of Liouville-Caputo integro-differential $\mathbb{B} \mathbb{V}$. Also, by assuming special values for coefficients, our problem is reduced to some simpler forms of the fractional boundary value problems. In other words, if $r=1$ and $\tau^{*}=1$, then Liouville-Caputo $\mathbb{B V} \mathbb{P}(3)$ is reduced to the following one:

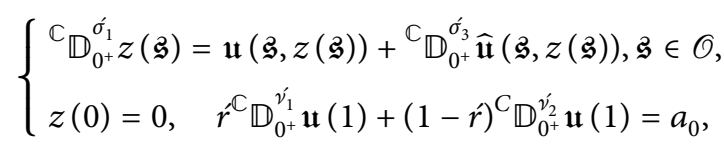

and if $\dot{r}=0$ and $\tau^{*}=1$, then Liouville-Caputo $\mathbb{B M P}$ (3) becomes

$$
\left\{\begin{array}{l}
\left(r^{C} \mathbb{D}_{0^{+}}^{\sigma_{1}^{\prime}}+\left(1-\dot{r}^{\prime}\right) \square_{0^{+}}^{\sigma^{\prime} \sigma_{2}}\right) z(\mathfrak{s})=\mathfrak{u}(\mathfrak{g}, z(\mathfrak{g}))+{ }^{C} \mathbb{D}_{0^{+}}^{\sigma_{3}} \widehat{\mathfrak{u}}(\mathfrak{g}, z(\mathfrak{g})), \quad \mathfrak{g} \in \mathcal{O}, \\
z(0)=0, \quad{ }^{C} \mathbb{D}_{0^{+}}^{\dot{v}_{2}} \mathfrak{u}(1)=a_{0} .
\end{array}\right.
$$

Precisely, and in comparison to some similar works, we have studied a more general problem in which we have illustrated our theoretical results by numerical examples and plots. These items make the novelty of our work because it is important for us that we can analyze the mentioned system analytically, numerically, and graphically. We also consider two different nonlinear terms in the right-hand side of the problem to cover a vast range of nonlinear functions arising in particular real fractional nonlinear mathematical models.

This paper is organized as follows. In Section 2, we recollect several assembled definitions of fractional calculus, useful lemmas, and some theorems about the fixed point that we need subsequently. Section 3 is divided into three parts. First, we utilize Banach's criterion of contraction mapping to establish our result regarding unique solution. In the next subsection, we give the proof of the first fundamental existence theorem of this paper by utilizing a fixed point criterion due to Krasnoselskii. Also, in the third subsection, we verify another result regarding existence theory with the aid of Leray-Schauder theorem. Along with these, appropriate applications in the framework of illustrative examples are provided, in which numerical simulation and the corresponding data are given in each part graphically. Finally, in Section 4, we point out the conclusions of our article.

\section{Preliminaries}

Before establishing our main results, we need to present some useful definitions and properties which help us to prove the essential lemmas and theorems.

Definition 1 (see [3]). Let $\sigma>0$ and $z:(0,+\infty) \longrightarrow \mathbb{R}$ be continuous. The integral

$$
\square_{0^{+}}^{\hat{\sigma}^{+}} z(\mathfrak{G})=\frac{1}{\Gamma(\hat{\sigma})} \int_{0}^{\mathfrak{F}}(\mathfrak{G}-\xi)^{\sigma^{\prime}-1} z(\xi) d \xi
$$

is named as the fractional integral in the Riemann-Liouville (FRL-integral) framework of order $\sigma$ provided this integral possesses a finite value.

Definition 2 (see [3]). Let $\dot{\sigma}>0, \quad \kappa=[\dot{\sigma}]+1$, and $\mathrm{z}:(0,+\infty) \longrightarrow \mathbb{R}$ belong to $\operatorname{AC}^{(\kappa)}((0, \infty), \mathbb{R})$. Then, the integral

$$
{ }^{C} \mathbb{D}_{0^{+}}^{\sigma^{\prime}} z(\mathfrak{S})=\frac{1}{\Gamma(\kappa-\sigma)} \int_{0}^{\mathfrak{S}}(\mathfrak{G}-\xi)^{\kappa-\sigma^{\prime}-1} z^{(\kappa)}(\xi) d \xi
$$

is named as the fractional derivative in the Caputo framework of order $\sigma$ provided its value is finite.

Remark 1. We have the following:

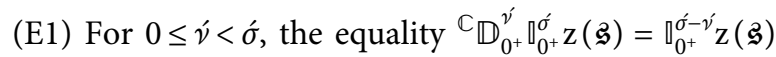
holds.

(E2) For $\dot{\sigma}>-1$ such that $\dot{\sigma} \neq \dot{v}-j,(j=1,2, \ldots, n)$, we have for $\mathfrak{g} \geq 0$,

$$
\mathbb{C}_{\mathbb{D}_{0^{+}}^{\sigma^{\prime}}} \mathfrak{g}^{v^{\prime}}=\frac{\Gamma(1+\hat{v})}{\Gamma(\hat{v}-\hat{\sigma}+1)} \mathfrak{g}^{v^{\prime}-\sigma^{\prime}} \text { and }{ }^{C} \mathbb{D}_{0^{+}}^{\sigma^{+}} \mathfrak{g}^{\nu^{\prime} j}=0, \quad(j=1,2, \ldots, n) .
$$

Proposition 1 (see [35]). Suppose that $z$ is contained in the space $\mathscr{L}(\mathcal{O}) \cap \mathscr{C}(\mathcal{O})$ and $\kappa=[\dot{\sigma}]+1$, where $\mathcal{O}=(0,1)$. Then,

$\square_{0^{+}}^{\sigma^{\prime}} C^{\mathbb{C}} \mathbb{D}_{0^{+}}^{\sigma^{\prime}} z(\mathfrak{g})=z(\mathfrak{g})+d_{1}+d_{2} \mathfrak{g}+d_{3} \mathfrak{g}^{2}+\cdots+d_{\kappa-1} \mathfrak{g}^{\kappa}$,

with $d_{1}, d_{2}, \ldots, d_{k-1} \in \mathbb{R}$. 
Lemma 1 (see [36]). Let $V$ be a nonempty, closed, and convex subset of a Banach space $\Xi$. Let $\Upsilon_{1}, \Upsilon_{2}$ be such that $\left(H_{i}\right) \Upsilon_{1} v+\Upsilon_{2} w \in V$ whenever $z, w \in V,\left(H_{i i}\right) \Upsilon_{1}$ is compact and continuous, and $\left(H_{i i i}\right) \Upsilon_{2}$ is contraction. Then, $\exists z \in V$ with $z=\Upsilon_{1} z+\Upsilon_{2} z$

Lemma 2 (see [37]). Let $\Xi$ be a Banach space, $V \subset \Xi$ be closed and convex in $\Xi, \mathscr{E} \subset V$ be open, $0 \in \mathscr{E}$, and let $\Theta: \overline{\mathscr{E}} \longrightarrow V$ be completely continuous. Then, either $\left(H_{i}\right)$ a fixed point is found for $\Theta$ in $\mathscr{E}$ or $\left(H_{i i}\right) \exists z \in \partial \mathrm{E}$ and $\ell \in \mathcal{O}$ with $z=\ell \Theta(z)$, where $\mathcal{O}=(0,1)$.

This key lemma will be useful for our study.

Lemma 3. Let $1<\hat{\sigma}_{1}, \hat{\sigma}_{3} \leq 2, \hat{\sigma}_{1}>\hat{\sigma}_{3}, \quad 0<\hat{\sigma}_{2} \leq 1, \quad 0<\dot{r} \leq 1$, $0 \leq \dot{r} \leq 1$, and $0<\hat{v}_{1}, \hat{v}_{2}<\hat{\sigma}_{1}-\hat{\sigma}_{3}$. Then, the integral equation

$$
\begin{aligned}
& z(\mathfrak{g})=\frac{\dot{r}-1}{r \Gamma\left(\hat{\sigma}_{1}+\dot{\sigma}_{2}\right)} \int_{0}^{\mathfrak{S}}(\mathfrak{g}-\xi)^{\hat{\sigma}_{1}+\dot{\sigma}_{2}-1} z(\xi) d \\
& +\frac{1}{r \Gamma\left(\hat{\sigma}_{1}\right)} \int_{0}^{\mathfrak{S}}(\mathfrak{g}-\xi)^{\sigma_{1}-1} \mathfrak{U}(\xi) d \xi \\
& +\frac{1}{r \Gamma\left(\hat{\sigma}_{1}-\hat{\sigma}_{3}\right)} \int_{0}^{\mathfrak{s}}(\mathfrak{B}-\xi)^{\sigma_{1}^{\prime}-\sigma_{3}-1} \widehat{\mathfrak{U}}(\xi) d \xi \\
& +\Pi \mathfrak{s}\left[a_{0}-\frac{\dot{r}(\dot{r}-1)}{r \Gamma\left(\dot{\sigma}_{1}+\dot{\sigma}_{2}-\dot{v}_{1}\right)} \int_{0}^{\tau^{*}}\left(\tau^{*}-\xi\right)^{\hat{\sigma}_{1}+\dot{\sigma}_{2}-\hat{\nu}_{1}-1} z(\xi) d \xi\right. \\
& -\frac{\dot{r}}{r \Gamma\left(\dot{\sigma}_{1}-\dot{v}_{1}\right)} \int_{0}^{\tau^{*}}\left(\tau^{*}-\xi\right)^{\sigma_{1}-\hat{v}_{1}^{\prime}-1} \mathfrak{U}(\xi) d \xi \\
& -\frac{\dot{r}}{r \Gamma\left(\dot{\sigma}_{1}-a_{0}\right)} \int_{0}^{\tau^{*}}\left(\tau^{*}-\xi\right)^{\sigma_{1}-\sigma_{3}-1} \widehat{\mathfrak{U}}(\xi) d \xi \\
& -\frac{(1-\dot{r})(\dot{r}-1)}{r \Gamma\left(\dot{\sigma}_{1}+\dot{\sigma}_{2}-\dot{v}_{2}\right)} \int_{0}^{\tau^{*}}\left(\tau^{*}-\xi\right)^{\hat{\sigma}_{1}+\dot{\sigma}_{2}-\dot{v}_{2}-1} z(\xi) d \xi \\
& -\frac{1-\dot{r}}{r \Gamma\left(\dot{\sigma}_{1}-\dot{v}_{2}\right)} \int_{0}^{\tau^{*}}\left(\tau^{*}-\xi\right)^{\dot{\sigma}_{1}-\dot{v}_{2}-1} \mathcal{U}(\xi) d \xi \\
& \left.-\frac{1-\dot{r}}{r \Gamma\left(\hat{\sigma}_{1}-\hat{\sigma}_{3}\right)} \int_{0}^{\tau^{*}}\left(\tau^{*}-\xi\right)^{\sigma_{1}-\dot{\sigma}_{3}-1} \widehat{\mathcal{U}}(\xi) d \xi\right]
\end{aligned}
$$

with

$$
\Pi=\left[\frac{\dot{r}}{\Gamma\left(2-\dot{v}_{1}\right)}+\frac{1-\dot{r}}{\Gamma\left(2-\dot{v}_{2}\right)}\right]^{-1},
$$

is the solution of the linear fractional $\mathbb{B M P}$ :

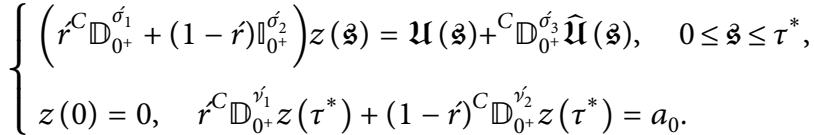

Proof. In view of the first equation of (12), we can write

$$
{ }^{C} \mathbb{D}_{0^{+}}^{\sigma_{1}} z(\mathfrak{G})=\frac{\dot{r}-1}{\dot{r}} \mathbb{D}_{0^{+}}^{\sigma_{2}} z(\mathfrak{G})+\frac{1}{\dot{r}} \mathfrak{U}(\mathfrak{G})+\frac{1_{C}}{\dot{r}} \mathbb{D}_{0^{+}}^{\sigma_{3}^{\prime}} \widehat{\mathfrak{U}}(\mathfrak{g}) .
$$

Taking the $\hat{\sigma}_{1}^{\text {th }}$ FRL-integral on (13), we find

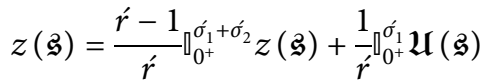

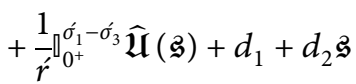

$$
\begin{aligned}
& =\frac{\dot{r}-1}{\dot{r} \Gamma\left(\dot{\sigma}_{1}+\dot{\sigma}_{2}\right)} \int_{0}^{\mathfrak{\mathcal { F }}}(\mathfrak{g}-\xi)^{\hat{\sigma}_{1}+\dot{\sigma}_{2}-1} z(\xi) d \xi \\
& +\frac{1}{r \Gamma\left(\dot{\sigma}_{1}\right)} \int_{0}^{\mathfrak{g}}(\mathfrak{G}-\xi)^{\sigma_{1}^{\prime}-1} \mathfrak{U}(\xi) d \xi \\
& +\frac{1}{r \Gamma\left(\dot{\sigma}_{1}-\sigma_{3}\right)} \\
& \int_{0}^{\mathfrak{S}}(\mathfrak{S}-\xi)^{\sigma_{1}-\sigma_{3}-1} \widehat{\mathfrak{U}}(\xi) d \xi+d_{1}+d_{2} \mathfrak{G}
\end{aligned}
$$

where $d_{1}, d_{2} \in \mathbb{R}$. The first boundary condition of (12) gives us $d_{1}=0$; then,

$$
\begin{aligned}
& z(\mathfrak{g})=\frac{\dot{r}-1}{\dot{r} \Gamma\left(\dot{\sigma}_{1}+\dot{\sigma}_{2}\right)} \int_{0}^{\mathfrak{g}}(\mathfrak{g}-\xi)^{\dot{\sigma}_{1}+\dot{\sigma}_{2}-1} z(\xi) d \xi \\
& +\frac{1}{r \Gamma\left(\dot{\sigma}_{1}\right)} \int_{0}^{\mathfrak{S}}(\mathfrak{S}-\xi)^{\hat{\sigma}_{1}-1} \mathfrak{U}(\xi) d \xi \\
& +\frac{1}{r \Gamma\left(\hat{\sigma}_{1}-\hat{\sigma}_{3}\right)} \int_{0}^{\mathfrak{3}}(\mathfrak{g}-\xi)^{\sigma_{1}-\sigma_{3}-1} \widehat{\mathfrak{U}}(\xi) d \xi+d_{2} \mathfrak{g},
\end{aligned}
$$

and by applying the $\eta^{\text {th }}$-Caputo derivative $\left(\eta \in\left\{\dot{v}_{1}, \dot{v}_{2}\right\}\right)$ with $0<\eta<\hat{\sigma}_{1}-\hat{\sigma}_{3}$ to (15), we obtain

$$
\begin{aligned}
& { }^{C} \mathbb{D}_{0^{+}}^{\eta} z(\mathfrak{g})=\frac{\dot{r}-1}{\dot{r} \Gamma\left(\hat{\sigma}_{1}+\hat{\sigma}_{2}-\eta\right)} \int_{0}^{\mathfrak{S}}(\mathfrak{G}-\xi)^{\hat{\sigma}_{1}+\tilde{\sigma}_{2}-\dot{\eta}^{-}-1} z(\mathfrak{g}) d \xi \\
& +\frac{1}{r \Gamma\left(\dot{\sigma}_{1}-\eta\right)} \int_{0}^{\mathfrak{S}}(\mathfrak{G}-\xi)^{\sigma_{1}^{\prime}-\dot{\eta}^{-1}} \mathfrak{U}(\xi) d \xi \\
& +\frac{1}{r \Gamma\left(\hat{\sigma}_{1}-\hat{\sigma}_{3}-\eta\right)} \int_{0}^{\mathfrak{3}}(\mathfrak{g}-\xi)^{\sigma_{1}-\hat{\sigma}_{3}-\dot{\eta}^{\prime}-1} \widehat{\mathfrak{U}}(\xi) d \xi \\
& +\frac{d_{2}}{\Gamma(2-\eta)} \mathfrak{g}^{1-\eta},
\end{aligned}
$$

because ${ }^{C} \mathbb{D}_{0^{+}}^{\eta} \mathfrak{g}=\Gamma(2) / \Gamma(2-\eta) \mathfrak{g}^{1-\dot{\eta}}$. Taking $\dot{\eta}=\dot{v}_{1}$ and $\dot{\eta}=$ $v_{2}$ in expression (16) and applying the second boundary condition of (12), we get 


$$
\begin{aligned}
& a_{0}=\frac{\dot{r}(\dot{r}-1)}{\dot{r} \Gamma\left(\hat{\sigma}_{1}+\dot{\sigma}_{2}-\dot{v}_{1}\right)} \int_{0}^{1}(1-\xi)^{\hat{\sigma}_{1}+\dot{\sigma}_{2}-\hat{\nu}_{1}-1} z(\mathfrak{g}) d \xi \\
& +\frac{\dot{r}}{r \Gamma\left(\dot{\sigma}_{1}-\dot{v}_{1}\right)} \int_{0}^{1}(1-\xi)^{\dot{\sigma}_{1}-\hat{v}_{1}^{\prime}-1} \mathfrak{U}(\xi) d \xi \\
& +\frac{\dot{r}}{r \Gamma\left(\dot{\sigma}_{1}-\dot{\sigma}_{3}-\dot{v}_{1}\right)} \int_{0}^{1}(1-\xi)^{\dot{\sigma}_{1}-\dot{\sigma}_{3}-\hat{v}_{1}-1} \widehat{\mathfrak{U}}(\xi) d \xi+\frac{d_{2} \dot{r}}{\Gamma\left(2-\dot{v}_{1}\right)} \\
& +\frac{(1-\dot{r})(\dot{r}-1)}{r \Gamma\left(\hat{\sigma}_{1}+\dot{\sigma}_{2}-\dot{v}_{2}\right)} \int_{0}^{1}(1-\xi)^{\hat{\sigma}_{1}+\dot{\sigma}_{2}-\dot{v}_{2}-1} z(\xi) d \xi \\
& +\frac{1-\dot{r}}{r \Gamma\left(\dot{\sigma}_{1}-\dot{v}_{2}\right)} \int_{0}^{1}(1-\xi)^{\sigma_{1}-v_{2}^{\prime}-1} \mathfrak{U}(\xi) d \xi \\
& +\frac{1-\dot{r}}{\dot{r} \Gamma\left(\hat{\sigma}_{1}-\dot{\sigma}_{3}-\dot{v}_{2}\right)} \int_{0}^{1}(1-\xi)^{\hat{\sigma}_{1}-\hat{\sigma}_{3}-\hat{v}_{2}-1} \widehat{\mathfrak{U}}(\xi) d \xi+\frac{d_{2}(1-\dot{r})}{\Gamma\left(2-\dot{v}_{2}\right)} .
\end{aligned}
$$

Therefore,

$$
\begin{aligned}
& d_{2}=\Pi\left[a_{0}-\frac{\dot{r}(\dot{r}-1)}{\dot{r} \Gamma\left(\dot{\sigma}_{1}+\dot{\sigma}_{2}-\dot{v}_{1}\right)} \int_{0}^{1}(1-\xi)^{\hat{\sigma}_{1}+\dot{\sigma}_{2}-\dot{v}_{1}-1} z(\xi) d \xi\right. \\
& -\frac{\dot{r}}{\dot{r} \Gamma\left(\beta_{1}-\dot{v}_{1}\right)} \int_{0}^{1}(1-\xi)^{\hat{\sigma}_{1}-\dot{v}_{1}-1} \mathcal{U}(\xi) d \xi \\
& -\frac{\dot{r}}{\dot{r} \Gamma\left(\hat{\sigma}_{1}-\hat{\sigma}_{3}-\dot{v}_{1}\right)} \int_{0}^{1}(1-\xi)^{\hat{\sigma}_{1}-\hat{\sigma}_{3}-\hat{v}_{1}-1} \widehat{\mathfrak{U}}(\xi) d \xi \\
& -\frac{(1-\dot{r})(\dot{r}-1)}{r \Gamma\left(\dot{\sigma}_{1}+\dot{\sigma}_{2}-\dot{v}_{2}\right)} \int_{0}^{1}(1-\xi)^{\dot{\sigma}_{1}+\hat{\sigma}_{2}-\hat{v}_{2}-1} z(\xi) d \xi \\
& -\frac{1-\dot{r}}{\dot{r} \Gamma\left(\dot{\sigma}_{1}-\dot{v}_{2}\right)} \int_{0}^{1}(1-\xi)^{\sigma_{1}^{\prime}-\dot{v}_{2}-1} \mathcal{U}(\xi) d \xi \\
& \left.-\frac{1-\dot{r}}{r \Gamma\left(\sigma_{1}-\dot{\sigma}_{3}-\dot{v}_{2}\right)} \int_{0}^{1}(1-\xi)^{\sigma_{1}-\sigma_{3}^{\prime}-\dot{v}_{2}-1} \widehat{\mathfrak{U}}(\xi) d \xi\right] .
\end{aligned}
$$

By substituting the value of $d_{2}$ in equation (15), we obtain integral equation (10). This ends the proof.

\section{Basic Theorems with Illustrative Examples}

Let $\mathcal{O}=[0,1]$ throughout the paper. Consider the Banach space $C(\mathcal{O}, \mathbb{R})$ of all continuous functions with the norm of uniform convergence

$$
\|z\|=\sup _{\mathfrak{s} \in \mathcal{O}}|z(\mathfrak{g})| .
$$

In accordance with Lemma 3 , it is obvious that we can transform our $\mathbb{B M P}(3)$ to the following fixed point problem $\mathrm{z}=P \mathrm{z}$, where $P$ is an operator $P: C(\mathcal{O}, \mathbb{R}) \longrightarrow C(\mathcal{O}, \mathbb{R})$ defined as

$$
\begin{aligned}
& P z(\mathfrak{g})=\frac{\dot{r}-1}{r \Gamma\left(\hat{\sigma}_{1}+\dot{\sigma}_{2}\right)} \int_{0}^{\mathfrak{g}}(\mathfrak{G}-\xi)^{\dot{\sigma}_{1}+\dot{\sigma}_{2}-1} z(\xi) d \xi \\
& =\frac{\dot{r}-1}{\dot{r} \Gamma\left(\dot{\sigma}_{1}+\dot{\sigma}_{2}\right)} \int_{0}^{\mathfrak{g}}(\mathfrak{g}-\xi)^{\dot{\sigma}_{1}+\dot{\sigma}_{2}-1} z(\xi) d \xi \\
& +\frac{1}{r \Gamma\left(\dot{\sigma}_{1}-\sigma_{3}\right)} \int_{0}^{\mathfrak{g}}(\mathfrak{g}-\xi)^{\sigma_{1}-\dot{\sigma}_{3}-1} \widehat{\mathfrak{u}}(\xi, z(\xi)) d \xi \\
& +\Pi \mathfrak{g}\left[a_{0}-\frac{\dot{r}(\dot{r}-1)}{r \Gamma\left(\hat{\sigma}_{1}+\dot{\sigma}_{2}-\dot{v}_{1}\right)} \int_{0}^{1}(1-\xi)^{\sigma_{1}^{\prime}+\dot{\sigma}_{2}-\hat{v}_{1}^{\prime}-1} z(\xi) d \xi\right. \\
& -\frac{\dot{r}}{\dot{r} \Gamma\left(\dot{\sigma}_{1}-\dot{v}_{1}\right)} \int_{0}^{1}(1-\xi)^{\sigma_{1}-\hat{v}_{1}^{\prime}-1} \mathfrak{u t}(\xi, z(\xi)) d \xi \\
& -\frac{\dot{r}}{r \Gamma\left(\hat{\sigma}_{1}-\dot{\sigma}_{3}-\dot{v}_{1}\right)} \int_{0}^{1}(1-\xi)^{\sigma_{1}^{\prime}-\dot{\sigma}_{3}-\dot{v}_{1}-1} \widehat{\mathfrak{u}}(\xi, z(\xi)) d \xi \\
& -\frac{(1-\dot{r})(\dot{r}-1)}{r \Gamma\left(\dot{\sigma}_{1}+\dot{\sigma}_{2}-\dot{v}_{2}\right)} \int_{0}^{1}(1-\xi)^{\dot{\sigma}_{1}+\dot{\sigma}_{2}-\dot{v}_{2}-1} z(\xi) d \xi \\
& -\frac{1-\dot{r}}{r \Gamma\left(\dot{\sigma}_{1}-\dot{v}_{2}\right)} \int_{0}^{1}(1-\xi)^{\sigma_{1}-\hat{v}_{2}-1} \mathfrak{u t}(\xi, z(\xi)) d \xi \\
& \left.-\frac{1-\dot{r}}{\dot{r} \Gamma\left(\dot{\sigma}_{1}-\hat{\sigma}_{3}-\dot{v}_{2}\right)} \int_{0}^{1}(1-\xi)^{\sigma_{1}-\hat{\sigma}_{3}-\dot{v}_{2}-1} \widehat{\mathfrak{u}}(\xi, z(\xi)) d \xi\right] \text {. }
\end{aligned}
$$

Therefore, $\mathbb{B M P}(3)$ admits a solution equivalent to saying that $P$ has a fixed point.

3.1. Banach Principle and Unique Solution. First, we apply Banach's principle of contraction mapping to prove our result of existence and uniqueness. To have computations with more convenience and clarity, we use these notations:

$$
\begin{aligned}
& \eta_{1}=\frac{1-\dot{r}}{\dot{r} \Gamma\left(\hat{\sigma}_{1}+\dot{\sigma}_{2}+1\right)}+\frac{\dot{r}(1-\dot{r}) \Pi}{\dot{r} \Gamma\left(\hat{\sigma}_{1}+\dot{\sigma}_{2}-\dot{v}_{1}+1\right)}+\frac{(1-\dot{r})(1-\dot{r}) \Pi}{r \Gamma\left(\hat{\sigma}_{1}+\dot{\sigma}_{2}-\dot{v}_{2}+1\right)}, \\
& \eta_{2}=\frac{1}{r \Gamma\left(\dot{\sigma}_{1}+1\right)}+\frac{r \Pi}{r \Gamma\left(\hat{\sigma}_{1}-\dot{v}_{1}+1\right)}+\frac{(1-\dot{r}) \Pi}{r \Gamma\left(\dot{\sigma}_{1}-\dot{v}_{2}+1\right)}, \\
& \eta_{3}=\frac{1}{r \Gamma\left(\hat{\sigma}_{1}-\hat{\sigma}_{3}+1\right)}+\frac{\dot{r} \Pi}{r \Gamma\left(\hat{\sigma}_{1}-\hat{\sigma}_{3}-\hat{v}_{1}+1\right)}+\frac{(1-\dot{r}) \Pi}{r \Gamma\left(\hat{\sigma}_{1}-\hat{\sigma}_{3}-\dot{v}_{2}+1\right)} .
\end{aligned}
$$


Theorem 1. Assume that $\mathfrak{u}, \widehat{\mathfrak{t}}: \mathcal{O} \times \mathbb{R} \longrightarrow \mathbb{R}$ are two continuous functions subject to the following two conditions:

$\left(\mathcal{S}_{1}\right):\left|\mathfrak{u}(\mathfrak{G}, z)-\mathfrak{u}\left(\mathfrak{g}, z^{\star}\right)\right| \leq \Theta_{1}\left|z-z^{\star}\right|$,

$\left(\mathcal{S}_{2}\right):\left|\widehat{\mathfrak{u}}(\mathfrak{G}, z)-\widehat{\mathfrak{u}}\left(\mathfrak{g}, z^{\star}\right)\right| \leq \Theta_{2}\left|z-z^{\star}\right|$,

for $\mathfrak{g} \in \mathcal{O}, z, z^{\star} \in \mathbb{R}$, where $\Theta_{1}, \Theta_{2}$ are two real positive constants. If

$$
\eta_{1}+\Theta_{1} \eta_{2}+\Theta_{2} \eta_{3}<1
$$

then the supposed $\mathbb{B M P}$ (3) admits a unique solution on $\mathcal{O}$.
Proof. By fixing $\mathfrak{u}^{\star}=\sup _{\mathfrak{z} \in \mathcal{O}}|\mathfrak{u}(\mathfrak{g}, 0)| \quad$ and $\widehat{\mathfrak{u}}^{\star}=\sup _{\mathfrak{g} \in \mathcal{O}}|\widehat{\mathfrak{u}}(\mathfrak{g}, 0)|$ with the choice $\bar{\Delta}_{1}>0$, so that

$$
\bar{\Delta}_{1} \geq \frac{\mathfrak{u}_{2}^{\star}+\widehat{\mathfrak{u}}^{\star} \eta_{3}+\Pi\left|a_{0}\right|}{1-\eta_{1}-\Theta_{1} \eta_{2}-\Theta_{2} \eta_{3}},
$$

where $\Pi$ is the positive constant expressed by (11), at first, we show that the image of the ball $\mathbb{B}_{\bar{\Delta}_{1}}$ by $P$ is included in $\mathbb{B}_{\bar{\Delta}_{1}}$, where

$$
\mathbb{B}_{\bar{\Delta}_{1}}=\left\{z \in C(\mathcal{O}, \mathbb{R}):\|z\| \leq \bar{\Delta}_{1}\right\} .
$$

So, for each $z \in \mathbb{B}_{\bar{\Delta}_{1}}$, we have

$$
\begin{aligned}
& |P z(\mathfrak{g})| \leq \frac{1-\dot{r}}{\dot{r} \Gamma\left(\dot{\sigma}_{1}+\dot{\sigma}_{2}\right)} \int_{0}^{1}(1-\xi)^{\hat{\sigma}_{1}+\dot{\sigma}_{2}-1}|z(\xi)| d \xi
\end{aligned}
$$

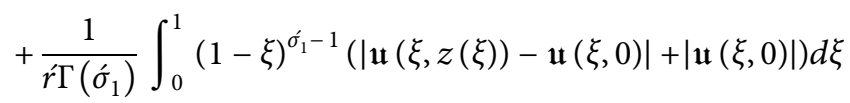

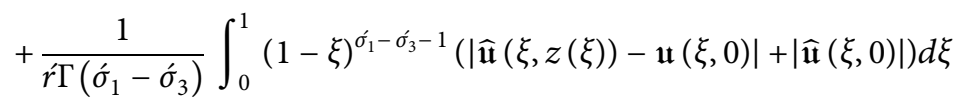

$$
\begin{aligned}
& +\Pi\left[\left|a_{0}\right|+\frac{\dot{r}(1-\dot{r})}{\dot{r} \Gamma\left(\dot{\sigma}_{1}+\dot{\sigma}_{2}-\dot{v}_{1}\right)} \int_{0}^{1}(1-\xi)^{\hat{\sigma}_{1}+\dot{\sigma}_{2}-\dot{v}_{1}-1}|z(\xi)| d \xi\right. \\
& +\frac{\dot{r}}{\dot{r} \Gamma\left(\dot{\sigma}_{1}-\dot{v}_{1}\right)} \int_{0}^{1}(1-\xi)^{\dot{\sigma}_{1}-\dot{v}_{1}-1}(|\mathfrak{u}(\xi, z(\xi))-\mathfrak{u}(\xi, 0)|+|\mathfrak{u}(\xi, 0)|) d \xi \\
& +\frac{\dot{r}}{r \Gamma\left(\dot{\sigma}_{1}-\dot{\sigma}_{3}-\dot{v}_{1}\right)} \int_{0}^{1}(1-\xi)^{\sigma_{1}-\dot{\sigma}_{3}-\dot{v}_{1}-1}(|\widehat{\mathfrak{u}}(\xi, z(\xi))-\widehat{\mathfrak{u}}(\xi, 0)|+|\widehat{\mathfrak{u}}(\xi, 0)|) d \xi \\
& +\frac{\left(1-\dot{r}^{\prime}\right)\left(1-\dot{r}^{\prime}\right)}{r \Gamma\left(\dot{\sigma}_{1}+\dot{\sigma}_{2}-\dot{v}_{2}\right)} \int_{0}^{1}(1-\xi)^{\sigma_{1}^{\prime}+\dot{\sigma}_{2}-\dot{v}_{2}-1}|z(\xi)| d \xi \\
& +\frac{1-\dot{r}}{r \Gamma\left(\dot{\sigma}_{1}-\dot{v}_{2}\right)} \int_{0}^{1}(1-\xi)^{\hat{\sigma}_{1}-\dot{v}_{2}-1}(|\mathfrak{u}(\xi, z(\xi))-\mathfrak{u}(\xi, 0)|+|\mathfrak{u}(\xi, 0)|) d \xi
\end{aligned}
$$

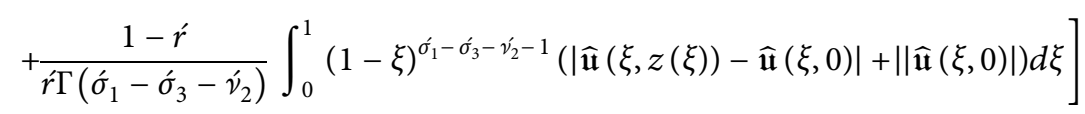

$$
\begin{aligned}
& \leq\|z\|\left[\frac{1-\dot{r}}{\dot{r} \Gamma\left(\dot{\sigma}_{1}+\dot{\sigma}_{2}+1\right)}+\frac{\dot{r}(1-\dot{r}) \Pi}{\dot{r} \Gamma\left(\dot{\sigma}_{1}+\dot{\sigma}_{2}-\dot{v}_{1}+1\right)}+\frac{(1-\dot{r})(1-\dot{r}) \Pi}{\dot{r} \Gamma\left(\dot{\sigma}_{1}+\dot{\sigma}_{2}-\dot{v}_{2}+1\right)}\right]
\end{aligned}
$$

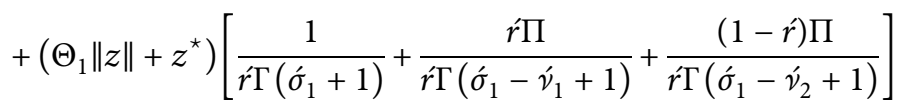

$$
\begin{aligned}
& +\left(\Theta_{2}\|z\|+z^{\star}\right)\left[\frac{1}{\dot{r} \Gamma\left(\hat{\sigma}_{1}-\hat{\sigma}_{3}+1\right)}+\frac{\dot{r} \Pi}{\dot{r} \Gamma\left(\hat{\sigma}_{1}-\hat{\sigma}_{3}-\dot{v}_{1}+1\right)}\right. \\
& \left.+\frac{(1-\dot{r}) \Pi}{\dot{r} \Gamma\left(\dot{\sigma}_{1}-\hat{\sigma}_{3}-\dot{v}_{2}+1\right)}\right]+\Pi\left|a_{0}\right| \\
& \leq \bar{\Delta}_{1}\left(\eta_{1}+\Theta_{1} \eta_{2}+\Theta_{2} \eta_{3}\right)+\mathfrak{u}^{\star} \eta_{2}+\widehat{\mathfrak{u}}^{\star} \eta_{3}+\Pi\left|a_{0}\right| \leq \bar{\Delta}_{1} .
\end{aligned}
$$


Journal of Function Spaces

7

This implies that $\|P z\| \leq \bar{\Delta}_{1}$. Thus $P\left(\mathbb{B}_{\bar{\Delta}_{1}}\right) \subset \mathbb{B}_{\bar{\Delta}_{1}}$. Next, for all $z, z^{\star} \in C(\mathcal{O}, \mathbb{R})$ and each $\mathfrak{G} \in \mathcal{O}$, we can write

$$
\begin{aligned}
& \left|P z(\mathfrak{g})-P z^{\star}(\mathfrak{g})\right| \leq \frac{1-\dot{r}}{\dot{r} \Gamma\left(\dot{\sigma}_{1}+\dot{\sigma}_{2}\right)} \int_{0}^{1}(1-\xi)^{\hat{\sigma}_{1}+\dot{\sigma}_{2}-1}\left|z(\xi)-z^{\star}(\xi)\right| d \xi \\
& +\frac{1}{r \Gamma\left(\sigma_{1}\right)} \int_{0}^{1}(1-\xi)^{\sigma_{1}-1}\left|\mathfrak{u}(\xi, z(\xi))-\mathfrak{u}\left(\xi, z^{\star}(\xi)\right)\right| d \xi \\
& +\frac{1}{r \Gamma\left(\sigma_{1}-\sigma_{3}\right)} \int_{0}^{1}(1-\xi)^{\sigma_{1}-\sigma_{3}^{\prime}-1}\left|\widehat{\mathfrak{u}}(\xi, z(\xi))-\widehat{\mathfrak{u}}\left(\xi, z^{\star}(\xi)\right)\right| d \xi \\
& +\Pi\left[\left|a_{0}\right|+\frac{\dot{r}(1-\dot{r})}{\dot{r} \Gamma\left(\dot{\sigma}_{1}+\dot{\sigma}_{2}-\dot{v}_{1}\right)} \int_{0}^{1}(1-\xi)^{\hat{\sigma}_{1}+\dot{\sigma}_{2}-\dot{v}_{1}-1}\left|z(\xi)-z^{\star}(\xi)\right| d \xi\right. \\
& +\frac{\dot{r}}{\dot{r} \Gamma\left(\dot{\sigma}_{1}-\dot{v}_{1}\right)} \int_{0}^{1}(1-\xi)^{\hat{\sigma}_{1}-\dot{v}_{1}-1}\left|\mathfrak{u}(\xi, z(\xi))-\mathfrak{u}\left(\xi, z^{\star}(\xi)\right)\right| d \xi \\
& +\frac{\dot{r}}{\dot{r} \Gamma\left(\dot{\sigma}_{1}-\dot{\sigma}_{3}-\dot{v}_{1}\right)} \int_{0}^{1}(1-\xi)^{\dot{\sigma}_{1}-\dot{\sigma}_{3}-\dot{v}_{1}-1}\left|\widehat{\mathfrak{u}}(\xi, z(\xi))-\widehat{\mathfrak{u}}\left(\xi, z^{\star}(\xi)\right)\right| d \xi \mathfrak{u} \\
& +\frac{\left(1-\dot{r}^{\prime}\right)\left(1-\dot{r}^{\prime}\right)}{\dot{r} \Gamma\left(\hat{\sigma}_{1}+\hat{\sigma}_{2}-\hat{v}_{2}\right)} \int_{0}^{1}(1-\xi)^{\hat{\sigma}_{1}+\hat{\sigma}_{2}-\hat{v}_{2}-1}\left|z(\xi)-z^{\star}(\xi)\right| d \xi \\
& +\frac{1-\dot{r}}{r \Gamma\left(\dot{\sigma}_{1}-\dot{v}_{2}\right)} \int_{0}^{1}(1-\xi)^{\sigma_{1}^{\prime}-\dot{v}_{2}-1}\left|\mathfrak{u}(\xi, z(\xi))-\mathfrak{u}\left(\xi, z^{\star}(\xi)\right)\right| d \xi \\
& \left.+\frac{1-\dot{r}}{\dot{r} \Gamma\left(\dot{\sigma}_{1}-\dot{\sigma}_{3}-\dot{v}_{2}\right)} \int_{0}^{1}(1-\xi)^{\sigma_{1}-\dot{\sigma}_{3}-\dot{v}_{2}-1}\left|\widehat{\mathfrak{u}}(\xi, z(\xi))-\widehat{\mathfrak{u}}\left(\xi, z^{\star}(\xi)\right)\right| d \xi\right] \\
& \leq\left\|z-z^{\star}\right\|\left[\frac{1-\dot{r}}{\dot{r} \Gamma\left(\dot{\sigma}_{1}+\dot{\sigma}_{2}+1\right)}+\frac{\dot{r}(1-\dot{r}) \Pi}{\dot{r} \Gamma\left(\dot{\sigma}_{1}+\dot{\sigma}_{2}-\dot{v}_{1}+1\right)}\right. \\
& \left.+\frac{\left(1-\hat{r}^{\prime}\right)(1-\hat{r}) \Pi}{r^{\prime} \Gamma\left(\hat{\sigma}_{1}+\dot{\sigma}_{2}-\dot{v}_{2}+1\right)}\right] \\
& +\Theta_{1}\left\|z-z^{\star}\right\|\left[\frac{1}{r \Gamma\left(\dot{\sigma}_{1}+1\right)}+\frac{r \Pi \Pi}{r \Gamma\left(\dot{\sigma}_{1}-\dot{v}_{1}+1\right)}\right. \\
& \left.+\frac{\left(1-\dot{r}^{\prime}\right) \Pi}{r \Gamma\left(\dot{\sigma}_{1}-\dot{v}_{2}+1\right)}\right] \\
& +\Theta_{2}\left\|z-z^{\star}\right\|\left[\frac{1}{r \Gamma\left(\dot{\sigma}_{1}-\hat{\sigma}_{3}+1\right)}+\frac{r^{\prime} \Pi}{r \Gamma\left(\dot{\sigma}_{1}-\dot{\sigma}_{3}-\dot{v}_{1}+1\right)}\right. \\
& \left.+\frac{(1-\dot{r}) \Pi}{\dot{r} \Gamma\left(\dot{\sigma}_{1}-\dot{\sigma}_{3}-\dot{v}_{2}+1\right)}\right]=\left[\eta_{1}+\Theta_{1} \eta_{2}+\Theta_{2} \eta_{3}\right]\left\|z-z^{\star}\right\| \text {, }
\end{aligned}
$$


which means that $\left\|P z-P z^{\star}\right\| \leq\left[\eta_{1}+\Theta_{1} \eta_{2}+\Theta_{2} \eta_{3}\right]\left\|z-z^{\star}\right\|$. Therefore, from (24), it follows that $P$ is a contraction. Consequently, the Banach principle of contraction mapping ensures that $P$ has a fixed point which represents the unique solution of our $\mathbb{B V P}$ (3). This ends the argument.

Example 1. Consider the Liouville-Caputo fractional $\mathbb{B} \mathbb{M}$

$$
\left\{\begin{array}{l}
\left(\frac{4}{5} C \mathbb{D}_{0^{+}}^{5 / 3}+\frac{1}{5} \mathbb{0}^{1 / 4}\right) z(\mathfrak{g}) \\
=\frac{2|z(\mathfrak{g})|}{(5+\mathfrak{g})^{2}(1+\exp (\mathfrak{I})|z(\mathfrak{I})|)}+{ }^{C} \mathbb{D}_{0^{+}}^{4 / 3}\left[\frac{\exp (-\mathfrak{g})|z(\mathfrak{I})|}{\pi^{2}+|z(\mathfrak{I})|}\right], \\
z(0)=0, \quad \frac{1}{4} C_{0^{+}} \mathbb{D}^{1 / 6} z(1)+\frac{3}{4} C_{0^{+}} \mathbb{D}^{1 / 2} z(1)=5 .
\end{array}\right.
$$

In the present example, we have $\hat{\sigma}_{1}=5 / 3 \in(1,2]$, $\hat{\sigma}_{2}=1 / 4 \in(0,1], \quad \hat{\sigma}_{3}=4 / 3 \in(1,2], \quad \dot{r}=4 / 5 \in(0,1]$, $\dot{r}=1 / 4 \in(0,1], \dot{v}_{1}=1 / 6 \in(0,1], \hat{v}_{2}=1 / 12 \in(0,1], a_{0}=5$, $\tau^{*}=1$, and

$\mathfrak{u}(\mathfrak{G}, \mathrm{z})=\frac{2|\mathrm{z}|}{(5+\mathfrak{g})^{2}(1+\exp (\mathfrak{g})|\mathrm{z}|)}, \quad \widehat{\mathfrak{u}}(\mathfrak{g}, \mathrm{z})=\frac{\exp (-\mathfrak{g})|\mathrm{z}|}{\pi^{2}+|\mathrm{z}|}$.

$$
\begin{aligned}
& \left|\mathfrak{u}(\mathfrak{G}, z)-\mathfrak{u}\left(\mathfrak{g}, z^{\star}\right)\right| \\
& =\left|\frac{2|z|}{(5+\mathfrak{g})^{2}(1+\exp (\mathfrak{G})|z|)}-\frac{2\left|z^{\star}\right|}{(5+\mathfrak{g})^{2}\left(1+\exp (\mathfrak{g})\left|z^{\star}\right|\right)}\right| \\
& =\frac{2}{(5+\mathfrak{G})^{2}}\left|\frac{|z|}{(1+\exp (\mathfrak{g})|z|)}-\frac{\left|z^{\star}\right|}{\left(1+\exp (\mathfrak{G})\left|z^{\star}\right|\right)}\right| \\
& \leq \frac{2}{25}\left|z-z^{\star}\right|, \\
& \left|\widehat{\mathfrak{u}}(\mathfrak{g}, z)-\widehat{\mathfrak{u}}\left(\mathfrak{g}, z^{\star}\right)\right| \\
& =\left|\frac{\exp (-\mathfrak{S})|z|}{\pi^{2}+|z|}-\frac{\exp (\mathfrak{S})\left|z^{\star}\right|}{\pi^{2}+\left|z^{\star}\right|}\right| \\
& \leq \frac{1}{\pi^{2}}\left|z-z^{\star}\right|,
\end{aligned}
$$

i.e., $\Theta_{1}=2 / 25 \approx 0.0800$ and $\Theta_{2}=1 / \pi^{2} \approx 0.1013$. A simple computation gives us

$\Pi=\left[\frac{\dot{r}}{\Gamma\left(2-\dot{v}_{1}\right)}+\frac{1-\dot{r}}{\Gamma\left(2-\dot{v}_{2}\right)}\right]^{-1}=\left[\frac{1 / 4}{\Gamma(2-(1 / 6))}+\frac{1-(4 / 5)}{\Gamma(2-(4 / 12))}\right]^{-1}$,

and

Then,

$$
\begin{aligned}
& \eta_{1}=\frac{1-\dot{r}}{r \Gamma\left(\hat{\sigma}_{1}+\dot{\sigma}_{2}+1\right)}+\frac{\dot{r}(1-\dot{r}) \Pi}{\dot{r} \Gamma\left(\hat{\sigma}_{1}+\hat{\sigma}_{2}-\dot{v}_{1}+1\right)}+\frac{(1-\dot{r})(1-\dot{r}) \Pi}{\dot{r} \Gamma\left(\hat{\sigma}_{1}+\dot{\sigma}_{2}-\dot{v}_{2}+1\right)} \\
& =\frac{1-4 / 5}{4 / 5 \Gamma(5 / 3+1 / 4+1)}+\frac{1 / 4(1-4 / 5) \Pi}{4 / 5 \Gamma(5 / 3+1 / 4-1 / 6+1)}+\frac{(1-1 / 4)(1-4 / 5) \Pi}{4 / 5 \Gamma(5 / 3+1 / 4-1 / 12+1)}, \\
& \eta_{2}=\frac{1}{r \Gamma\left(\hat{\sigma}_{1}+1\right)}+\frac{r \Pi}{r \Gamma\left(\hat{\sigma}_{1}-\hat{v}_{1}+1\right)}+\frac{(1-\dot{r}) \Pi}{\dot{r} \Gamma\left(\hat{\sigma}_{1}-\dot{v}_{2}+1\right)} \\
& =\frac{1}{4 / 5 \Gamma(5 / 3+1)}+\frac{1 / 4 \Pi}{4 / 5 \Gamma(5 / 3-1 / 6+1)}+\frac{(1-1 / 4) \Pi}{4 / 5 \Gamma(5 / 3-1 / 12+1)}, \\
& \eta_{3}=\frac{1}{r \Gamma\left(\hat{\sigma}_{1}-\hat{\sigma}_{3}+1\right)}+\frac{\hat{r} \Pi}{\dot{r} \Gamma\left(\hat{\sigma}_{1}-\hat{\sigma}_{3}-\hat{v}_{1}+1\right)}+\frac{(1-\dot{r}) \Pi}{\dot{r} \Gamma\left(\hat{\sigma}_{1}-\hat{\sigma}_{3}-\hat{v}_{2}+1\right)} \\
& =\frac{1}{4 / 5 \Gamma(5 / 3-4 / 3+1)}+\frac{1 / 4 \Pi}{4 / 5 \Gamma(5 / 3-4 / 3-1 / 6+1)}+\frac{(1-1 / 4) \Pi}{4 / 5 \Gamma(5 / 3-4 / 3-1 / 12+1)} .
\end{aligned}
$$

So, $\quad \Pi \approx 0.9607, \quad \eta_{1} \approx 0.2766, \quad \eta_{1} \approx 1.6945, \quad$ and $\eta_{3} \approx 2.7171$ which lead to

$$
\eta_{1}+\Theta_{1} \eta_{2}+\Theta_{2} \eta_{3} \approx 0.6875<1 .
$$

Table 1 shows these results. These values are plotted in Figure 1. By using the result of Theorem 1, we conclude that our BVP (29) admits only one solution on $[0,1]$.
3.2. Existence Result Based on Krasnoselskii's Criterion. Our existence analysis in this part is a consequence of Krasnoselskii's criterion (Lemma 1). For this fact, we introduce two operators $P_{1}$ and $P_{2}$ defined on the ball

$$
\mathbb{B}_{\bar{\Delta}_{2}}=\left\{z \in C(\mathcal{O}, \mathbb{R}):\|z\| \bar{\Delta}_{2}\right\},
$$

such that, for all $\mathfrak{g} \in \mathcal{O}$, 
TABLE 1: Numerical values of $\eta_{1}, \eta_{2}, \eta_{3}$, and $\Pi$, for $\dot{r} \in(0,1]$, in Example 1 .

\begin{tabular}{|c|c|c|c|c|c|}
\hline \multirow[b]{2}{*}{$\dot{r}$} & \multicolumn{5}{|c|}{$\dot{r} \in(0,1]$} \\
\hline & $\Pi$ & $\eta_{1}$ & $\eta_{2}$ & $\eta_{3}$ & $\eta_{1}+\Theta_{1} \eta_{2}+\Theta_{2} \eta_{3}$ \\
\hline 0.05 & 0.9607 & 21.0209 & 27.1126 & 43.4734 & 27.5946 \\
\hline 0.10 & 0.9607 & 9.9572 & 13.5563 & 21.7367 & 13.2441 \\
\hline 0.15 & 0.9607 & 6.2694 & 9.0375 & 14.4911 & 8.4606 \\
\hline 0.20 & 0.9607 & 4.4254 & 6.7781 & 10.8684 & 6.0689 \\
\hline 0.25 & 0.9607 & 3.3191 & 5.4225 & 8.6947 & 4.6338 \\
\hline 0.30 & 0.9607 & 2.5815 & 4.5188 & 7.2456 & 3.6771 \\
\hline 0.35 & 0.9607 & 2.0547 & 3.8732 & 6.2105 & 2.9938 \\
\hline 0.40 & 0.9607 & 1.6595 & 3.3891 & 5.4342 & 2.4813 \\
\hline 0.45 & 0.9607 & 1.3522 & 3.0125 & 4.8304 & 2.0826 \\
\hline 0.50 & 0.9607 & 1.1064 & 2.7113 & 4.3473 & 1.7637 \\
\hline 0.55 & 0.9607 & 0.9052 & 2.4648 & 3.9521 & 1.5028 \\
\hline 0.60 & 0.9607 & 0.7376 & 2.2594 & 3.6228 & 1.2854 \\
\hline 0.65 & 0.9607 & 0.5957 & 2.0856 & 3.3441 & 1.1014 \\
\hline 0.70 & 0.9607 & 0.4742 & 1.9366 & 3.1052 & 0.9437 \\
\hline 0.75 & 0.9607 & 0.3688 & 1.8075 & 2.8982 & 0.8070 \\
\hline 0.80 & 0.9607 & 0.2766 & 1.6945 & 2.7171 & 0.6875 \\
\hline 0.85 & 0.9607 & 0.1952 & 1.5949 & 2.5573 & 0.5819 \\
\hline 0.90 & 0.9607 & 0.1229 & 1.5063 & 2.4152 & 0.4881 \\
\hline 0.95 & 0.9607 & 0.0582 & 1.4270 & 2.2881 & 0.4042 \\
\hline
\end{tabular}

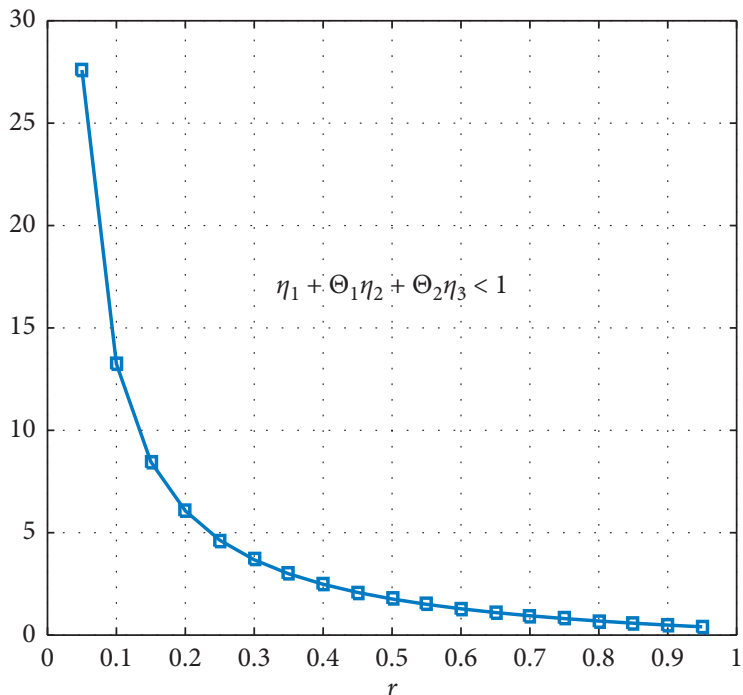

(a)

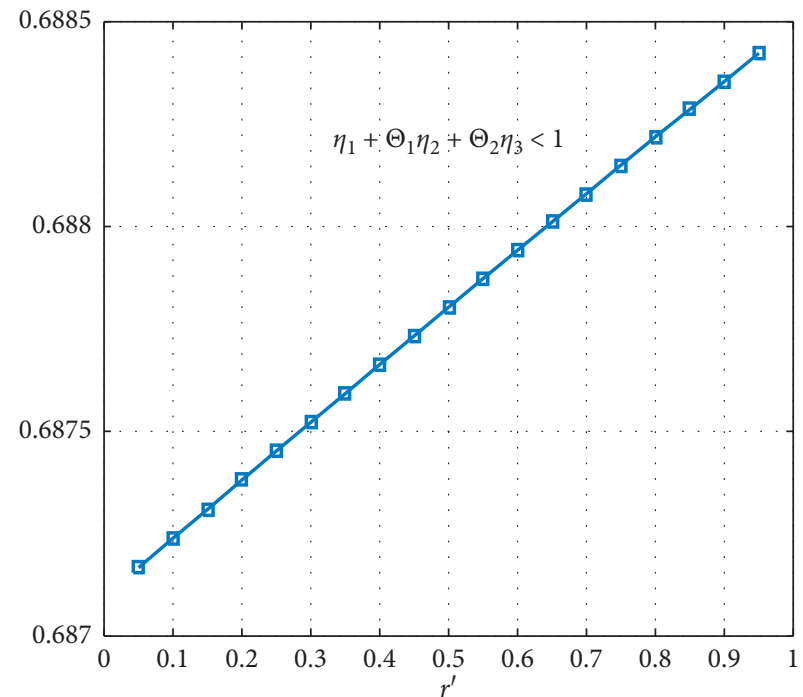

(b)

Figure 1: Graphical representation of $\eta_{1}+\Theta_{1} \eta_{2}+\Theta_{2} \eta_{3}$ in Example 1. (a) $r \in(0,1], \dot{r}=1 / 4$. (b) $r=4 / 5 \dot{r} \in(0,1]$. 


$$
\begin{aligned}
& P_{1} z(\mathfrak{g})=\frac{\dot{r}-1}{\dot{r} \Gamma\left(\hat{\sigma}_{1}+\hat{\sigma}_{2}\right)} \int_{0}^{\mathfrak{S}}(\mathfrak{g}-\xi)^{\hat{\sigma}_{1}+\hat{\sigma}_{2}-1} z(\xi) d \xi \\
& -\frac{\Pi \mathfrak{s} \dot{r}(\dot{r}-1)}{r \Gamma\left(\dot{\sigma}_{1}+\dot{\sigma}_{2}-\dot{v}_{1}\right)} \int_{0}^{1}(1-\xi)^{\sigma_{1}+\dot{\sigma}_{2}-\hat{\nu}_{1}-1} z(\xi) d \xi \\
& -\frac{\Pi \mathfrak{F}(1-\dot{r})(\dot{r}-1)}{\dot{r} \Gamma\left(\dot{\sigma}_{1}+\dot{\sigma}_{2}-\dot{v}_{2}\right)} \int_{0}^{1}(1-\xi)^{\dot{\sigma}_{1}+\dot{\sigma}_{2}-\hat{v}_{2}-1} z(\xi) d \xi,
\end{aligned}
$$

and

$$
\begin{aligned}
& P_{2} z(\mathfrak{g})=\frac{1}{\dot{r} \Gamma\left(\dot{\sigma}_{1}\right)} \int_{0}^{\mathfrak{S}}(\mathfrak{g}-\xi)^{\sigma_{1}-1} \mathfrak{u}(\xi, z(\xi)) d \xi \\
& +\frac{1}{r \Gamma\left(\hat{\sigma}_{1}-\hat{\sigma}_{3}\right)} \int_{0}^{\mathfrak{3}}(\mathfrak{g}-\xi)^{\sigma_{1}-\sigma_{3}-1} \widehat{\mathfrak{u}}(\xi, z(\xi)) d \xi \\
& +\Pi \mathfrak{g}\left[a_{0}-\frac{\dot{r}}{\dot{r} \Gamma\left(\dot{\sigma}_{1}-\dot{v}_{1}\right)} \int_{0}^{1}(1-\xi)^{\dot{\sigma}_{1}-\hat{v}_{1}-1} \mathfrak{u}(\xi, z(\xi)) d \xi\right. \\
& -\frac{\dot{r}}{\dot{r} \Gamma\left(\dot{\sigma}_{1}-\dot{\sigma}_{3}-\dot{v}_{1}\right)} \int_{0}^{1}(1-\xi)^{\sigma_{1}-\dot{\sigma}_{3}-\dot{v}_{1}-1} \widehat{\mathfrak{u}}(\xi, z(\xi)) d \xi \\
& -\frac{1-\dot{r}}{\dot{r} \Gamma\left(\beta_{1}-\dot{v}_{2}\right)} \int_{0}^{1}(1-\xi)^{\sigma_{1}-\dot{v}_{2}-1} \mathfrak{u}(\xi, z(\xi)) d \xi \\
& \left.-\frac{1-\dot{r}}{r \Gamma\left(\sigma_{1}-\dot{\sigma}_{3}-v_{2}\right)} \int_{0}^{1}(1-\xi)^{\sigma_{1}-\hat{\sigma}_{3}-\hat{v}_{2}-1} \widehat{\mathfrak{u}}(\xi, z(\xi)) d \xi\right] \text {. }
\end{aligned}
$$

Theorem 2. Consider the continuous functions $\mathfrak{u}, \widehat{\mathfrak{t}}: \mathcal{O} \times \mathbb{R} \longrightarrow \mathbb{R}$ which, respectively, satisfy the conditions $\left(\mathcal{S}_{1}\right)$ and $\left(\mathcal{S}_{2} 2\right)$ of Theorem 1. Furthermore, suppose that

$$
\begin{aligned}
& \left(\mathcal{S}_{3}\right):|\mathfrak{i}(\mathfrak{H}, z)| \leq \varrho_{1}(\mathfrak{G}), \\
& \left(\mathcal{S}_{4}\right):|\widehat{\mathfrak{u}}(\mathfrak{g}, z)| \leq \varrho_{2}(\mathfrak{I}),
\end{aligned}
$$

for $(\mathfrak{g}, z) \in \mathcal{O} \times \mathbb{R}$, and $\varrho_{j} \in C\left(\mathcal{O}, \mathbb{R}^{+}\right), j=1,2$. If $\eta_{1}<1$ which is defined in equation (21), then the supposed $\mathbb{B M} \mathbb{P}$ (3) admits at least one solution defined on $\mathcal{O}$.

Proof. Put $\left\|\varrho_{j}\right\|=\sup _{\mathfrak{s} \in \mathcal{O}}\left|\varrho_{j}(\mathfrak{g})\right|,(j=1,2)$. We choose $\bar{\Delta}_{2}$ so that

$$
\bar{\Delta}_{2} \geq \frac{\left\|\varrho_{1}\right\| \eta_{2}+\left\|\varrho_{2}\right\| \eta_{3}+\Pi\left|a_{0}\right|}{1-\eta_{1}} .
$$

In the first place, we prove that $P_{1} \mathrm{z}+P_{2} \mathrm{z}^{\star} \in B_{\bar{\Delta}_{2}}$. So, for all $\mathrm{z}, \mathrm{z}^{\star} \in B_{\bar{\Delta}_{2}}$, we have

$$
\begin{aligned}
& \left|P_{1} z(\mathfrak{g})+P_{2} z^{\star}(\mathfrak{g})\right| \leq \frac{1-\dot{r}}{r \Gamma\left(\dot{\sigma}_{1}+\dot{\sigma}_{2}\right)} \int_{0}^{1}(1-\xi)^{\hat{\sigma}_{1}+\dot{\sigma}_{2}-1}|z(\xi)| d \xi \\
& +\frac{1}{r \Gamma\left(\dot{\sigma}_{1}\right)} \int_{0}^{1}(1-\xi)^{\sigma_{1}-1}\left|\mathfrak{u}\left(\xi, z^{\star}(\xi)\right)\right| d \xi \\
& +\frac{\Pi \dot{r}(1-\dot{r})}{r \Gamma\left(\dot{\sigma}_{1}+\dot{\sigma}_{2}-\dot{v}_{1}\right)} \int_{0}^{1}(1-\xi)^{\hat{\sigma}_{1}+\dot{\sigma}_{2}-\dot{\nu}_{1}-1}|z(\xi)| d \xi \\
& +\frac{\Pi(1-\dot{r})(1-\dot{r})}{r \Gamma\left(\dot{\sigma}_{1}+\dot{\sigma}_{2}-\dot{v}_{2}\right)} \int_{0}^{1}(1-\xi)^{\hat{\sigma}_{1}+\dot{\sigma}_{2}-\hat{v}_{2}-1}|z(\xi)| d \xi
\end{aligned}
$$

$$
\begin{aligned}
& +\frac{1}{r \Gamma\left(\dot{\sigma}_{1}-\dot{\sigma}_{3}\right)} \int_{0}^{1}(1-\xi)^{\hat{\sigma}_{1}-\hat{\sigma}_{3}-1}\left|\widehat{\mathfrak{u}}\left(\xi, z^{\star}(\xi)\right)\right| d \xi \\
& +\Pi\left[\left|a_{0}\right|+\frac{\dot{r}}{\dot{r} \Gamma\left(\dot{\sigma}_{1}-\dot{v}_{1}\right)} \int_{0}^{1}(1-\xi)^{\dot{\sigma}_{1}-\dot{v}_{1}-1}\left|\mathfrak{u}\left(\xi, z^{\star}(\xi)\right)\right| d \xi\right. \\
& +\frac{\dot{r}}{r \Gamma\left(\dot{\sigma}_{1}-\dot{\sigma}_{3}-\dot{v}_{1}\right)} \int_{0}^{1}(1-\xi)^{\sigma_{1}-\dot{\sigma}_{3}-\dot{\nu}_{1}-1}\left|\widehat{\mathfrak{u}}\left(\xi, z^{\star}(\xi)\right)\right| d \xi \\
& +\frac{1-\dot{r}}{\dot{r} \Gamma\left(\dot{\sigma}_{1}-\dot{v}_{2}\right)} \int_{0}^{1}(1-\xi)^{\hat{\sigma}_{1}-\hat{v}_{2}-1}\left|\mathfrak{u}\left(\xi, z^{\star}(\xi)\right)\right| d \xi \\
& \left.+\frac{1-\dot{r}}{r \Gamma\left(\dot{\sigma}_{1}-\dot{\sigma}_{3}-\dot{v}_{2}\right)} \int_{0}^{1}(1-\xi)^{\sigma_{1}-\sigma_{3}-\dot{v}_{2}-1}\left|\widehat{\mathfrak{u}}\left(\xi, z^{\star}(\xi)\right)\right| d \xi\right] \\
& \leq\|z\|\left[\frac{1-\dot{r}}{\dot{r} \Gamma\left(\dot{\sigma}_{1}+\dot{\sigma}_{2}+1\right)}+\frac{\dot{r}(1-\dot{r}) \Pi}{\dot{r} \Gamma\left(\dot{\sigma}_{1}+\dot{\sigma}_{2}-\dot{v}_{1}+1\right)}\right. \\
& \left.+\frac{\left(1-\dot{r}^{\prime}\right)(1-\dot{r}) \Pi}{\dot{r} \Gamma\left(\dot{\sigma}_{1}+\dot{\sigma}_{2}-\dot{v}_{2}+1\right)}\right] \\
& +\left\|\varrho_{1}\right\|\left[\frac{1}{r \Gamma\left(\hat{\sigma}_{1}+1\right)}+\frac{\dot{r} \Pi}{r \Gamma\left(\hat{\sigma}_{1}-\dot{v}_{1}+1\right)}+\frac{(1-\dot{r}) \Pi}{r \Gamma\left(\hat{\sigma}_{1}-\dot{v}_{2}+1\right)}\right] \\
& +\left\|\varrho_{2}\right\|\left[\frac{1}{r \Gamma\left(\dot{\sigma}_{1}-\hat{\sigma}_{3}+1\right)}+\frac{\dot{r} \Pi}{r \Gamma\left(\dot{\sigma}_{1}-\dot{\sigma}_{3}-\dot{v}_{1}+1\right)}\right. \\
& \left.+\frac{(1-\dot{r}) \Pi}{r \Gamma\left(\sigma_{1}-\dot{\sigma}_{3}-\dot{v}_{2}+1\right)}\right]+\Pi\left|a_{0}\right| \\
& \leq \bar{\Delta}_{2} \eta_{1}+\left\|\varrho_{1}\right\| \eta_{2}+\left\|\varrho_{2}\right\| \eta_{3}+\Pi\left|a_{0}\right| \leq \bar{\Delta}_{2} \text {. }
\end{aligned}
$$

Thus, $\quad\left\|P_{1} \mathrm{z}+P_{2} \mathrm{z}^{\star}\right\| \leq \bar{\Delta}_{2}$, which means that $P_{1} \mathrm{z}+P_{2} \mathrm{z}^{\star} \in B_{\overline{\Delta_{2}}}$. Now, we establish that $P_{1}$ is a contraction. For $\mathrm{z}, \mathrm{z}^{\star} \in B_{\bar{\Delta}_{2}}$, we can write

$$
\begin{aligned}
& \left|P_{1} z(\mathfrak{g})-P_{1} z^{\star}(\mathfrak{g})\right| \leq \frac{1-\dot{r}}{r \Gamma\left(\dot{\sigma}_{1}+\dot{\sigma}_{2}\right)} \\
& \int_{0}^{1}(1-\xi)^{\sigma_{1}+\dot{\sigma}_{2}-1}\left|z(\xi)-z^{\star}(\xi)\right| d \xi \\
& +\frac{\Pi \dot{r}(1-\dot{r})}{\dot{r} \Gamma\left(\dot{\sigma}_{1}+\dot{\sigma}_{2}-\dot{v}_{1}\right)} \int_{0}^{1}(1-\xi)^{\sigma_{1}+\dot{\sigma}_{2}-\dot{v}_{1}-1}\left|z(\xi)-z^{\star}(\xi)\right| d \xi \\
& +\frac{\Pi(1-\dot{r})(1-\dot{r})}{\dot{r} \Gamma\left(\dot{\sigma}_{1}+\dot{\sigma}_{2}-\dot{v}_{2}\right)} \int_{0}^{1}(1-\xi)^{\hat{\sigma}_{1}+\hat{\sigma}_{2}-\hat{v}_{2}-1}\left|z(\xi)-z^{\star}(\xi)\right| d \xi \\
& \leq\left\|z-z^{\star}\right\|\left[\frac{1-\dot{r}}{\dot{r} \Gamma\left(\dot{\sigma}_{1}+\dot{\sigma}_{2}+1\right)}+\frac{\dot{r}(1-\dot{r}) \Pi}{\dot{r} \Gamma\left(\dot{\sigma}_{1}+\dot{\sigma}_{2}-\dot{v}_{1}+1\right)}\right. \\
& \left.+\frac{\left(1-\dot{r}^{\prime}\right)\left(1-\dot{r}^{\prime}\right) \Pi}{r \Gamma\left(\dot{\sigma}_{1}+\dot{\sigma}_{2}-\dot{v}_{2}+1\right)}\right]=\eta_{1}\left\|z-z^{\star}\right\| \text {. }
\end{aligned}
$$

Then,

$$
\left\|P_{1} z-P_{1} z^{\star}\right\| \leq \eta_{1}\left\|z-z^{\star}\right\|
$$

From the condition $\eta_{1}<1$, it follows that $P_{1}$ is a contraction mapping. On the other hand, we know that the continuity of $P_{2}$ occurs immediately from that of the functions $\mathfrak{t}$ and $\widehat{\mathfrak{t}}$. Also, it is simple to establish that for $\mathrm{z} \in B_{\bar{\Delta}_{2}}$, 


$$
\left\|P_{2} z\right\| \leq\left\|\varrho_{1}\right\| \eta_{2}+\left\|\varrho_{2}\right\| \eta_{3} .
$$

In other words, $P_{2}$ is uniformly bounded on $B_{\overline{\Delta_{2}}}$. In this moment, we need to show that $P_{2}$ is equicontinuous. Let $\mathfrak{u}^{\star}=\sup _{(\mathfrak{g}, z) \in \mathcal{O} \times \mathbb{R}}|\mathfrak{u t}(\mathfrak{g}, z)|$, and $\widehat{\mathfrak{u}}^{\star}=\sup _{(\mathfrak{g}, z) \in \mathcal{O} \times \mathbb{R}}|\widehat{\mathfrak{u}}(\mathfrak{g}, z)|$.

This allows us to write, for any $\left(\mathfrak{g}_{1}, \mathfrak{B}_{2}\right) \in \mathcal{O} \times \mathcal{O}$, where $\left(\mathfrak{g}_{1}<\mathfrak{g}_{2}\right)$, and for all $\mathrm{z} \in B_{\overline{\Delta_{2}}}$ :

$\left|P_{2} \mathfrak{u}\left(\mathfrak{g}_{2}\right)-P_{2} \mathfrak{t}\left(\mathfrak{g}_{1}\right)\right|=\frac{1}{r \Gamma\left(\hat{\sigma}_{1}\right)}\left[\int_{0}^{\mathfrak{g}_{2}}\left(\mathfrak{g}_{2}-\xi\right)^{\sigma_{1}^{\prime}-1} \mathfrak{u}(\xi, z(\xi)) d \xi\right.$

$\left.-\int_{0}^{\mathfrak{G}_{1}}\left(\mathfrak{B}_{1}-\xi\right)^{\hat{\sigma}_{1}-1} \mathfrak{u}(\xi, z(\xi)) d \xi\right]$

$+\frac{1}{r \Gamma\left(\hat{\sigma}_{1}-\hat{\sigma}_{3}\right)}\left[\int_{0}^{\mathfrak{g}_{2}}\left(\mathfrak{g}_{2}-\xi\right)^{\hat{\sigma}_{1}-\dot{\sigma}_{3}-1} \widehat{\mathfrak{u}}(\xi, z(\xi)) d \xi\right.$

$\left.-\int_{0}^{\mathfrak{S}_{1}}\left(\mathfrak{I}_{1}-\xi\right)^{\hat{\sigma}_{1}-\hat{\sigma}_{3}-1} \widehat{\mathfrak{u}}(\xi, z(\xi)) d \xi\right]+\Pi\left(\mathfrak{\mathfrak { S }}_{2}-\mathfrak{I}_{1}\right)\left[a_{0}\right.$

$-\frac{\dot{r}}{r \Gamma\left(\hat{\sigma}_{1}-\dot{v}_{1}\right)} \int_{0}^{1}(1-\xi)^{\sigma_{1}^{\prime}-\dot{v}_{1}-1} \mathfrak{u}(\xi, z(\xi)) d \xi$

$-\frac{\dot{r}}{r \Gamma\left(\dot{\sigma}_{1}-\dot{\sigma}_{3}-\dot{v}_{1}\right)} \int_{0}^{1}(1-\xi)^{\sigma_{1}-\dot{\sigma}_{3}^{\prime}-\hat{\nu}_{1}^{\prime}-1} \widehat{\mathfrak{u}}(\xi, z(\xi)) d \xi$

$-\frac{1-\dot{r}}{r \Gamma\left(\hat{\sigma}_{1}-\dot{v}_{2}\right)} \int_{0}^{1}(1-\xi)^{\hat{\sigma}_{1}-\hat{v}_{2}-1} \mathfrak{u}(\xi, z(\xi)) d \xi$

$\left.-\frac{1-\dot{r}}{r \Gamma\left(\hat{\sigma}_{1}-\hat{\sigma}_{3}-\hat{v}_{2}\right)} \int_{0}^{1}(1-\xi)^{\hat{\sigma}_{1}-\hat{\sigma}_{3}^{\prime}-\hat{\nu}_{2}^{\prime}-1} \widehat{\mathfrak{u}}(\xi, z(\xi)) d \xi\right]$

$\leq \frac{\mathfrak{t}^{\star}}{r \Gamma\left(\hat{\sigma}_{1}\right)}\left[\int_{0}^{\mathfrak{g}_{1}}\left[\left(\mathfrak{g}_{2}-\xi\right)^{\hat{\sigma}_{1}-1}-\left(\mathfrak{g}_{1}-\xi\right)^{\hat{\sigma}_{1}-1}\right] d \xi\right.$

$\left.+\int_{\mathfrak{S}_{1}}^{\mathfrak{g}_{2}}\left(\mathfrak{I}_{2}-\xi\right)^{\dot{\sigma}_{1}-1} d \xi\right]$

$+\frac{\widehat{\mathfrak{u}}^{\star}}{r \Gamma\left(\hat{\sigma}_{1}-\hat{\sigma}_{3}\right)}\left[\int_{0}^{\mathfrak{g}_{1}}\left[\left(\mathfrak{\mathfrak { I }}_{2}-\xi\right)^{\hat{\sigma}_{1}-\dot{\sigma}_{3}-1}-\left(\mathfrak{\mathfrak { B }}_{1}-\xi\right)^{\hat{\sigma}_{1}-\hat{\sigma}_{3}-1}\right] d \xi\right.$

$\left.+\int_{\mathfrak{B}_{1}}^{\mathfrak{g}_{2}}\left(\mathfrak{g}_{2}-\xi\right)^{\hat{\sigma}_{1}-\dot{\sigma}_{3}-1} d \xi\right]$

$$
\begin{aligned}
& +\Pi\left(\mathfrak{g}_{2}-\mathfrak{g}_{1}\right)\left[\left|a_{0}\right|+\frac{\dot{r} \nu^{\star}}{r \Gamma\left(\hat{\sigma}_{1}-\dot{\nu}_{1}\right)} \int_{0}^{1}(1-\xi)^{\dot{\sigma}_{1}-\dot{\nu}_{1}-1} d \xi\right. \\
& +\frac{\dot{r} \hat{\mathfrak{u}}^{\star}}{r \Gamma\left(\hat{\sigma} \hat{\sigma}_{1}-\hat{\sigma}_{3}-\hat{v}_{1}\right)} \int_{0}^{1}(1-\xi)^{\hat{\sigma}_{1}-\hat{\sigma}_{3}-\hat{\nu}_{1}^{\prime}-1} d \xi
\end{aligned}
$$

$$
\begin{aligned}
& +\frac{(1-\hat{r}) \mathfrak{u t}^{\star}}{r \Gamma\left(\hat{\sigma}_{1}-\hat{v}_{2}\right)} \int_{0}^{1}(1-\xi)^{\hat{\sigma}_{1}-\hat{v}_{2}-1} d \xi \\
& \left.+\frac{(1-\hat{r}) \widehat{\mathfrak{u}}^{*}}{r \Gamma\left(\hat{\sigma}_{1}-\hat{\sigma}_{3}-\hat{v}_{2}\right)} \int_{0}^{1}(1-\xi)^{\hat{\sigma}_{1}-\hat{\sigma}_{3}-\hat{v}_{2}-1} d \xi\right] \\
& \leq \frac{\mathfrak{u}^{\star}}{r \Gamma\left(\hat{\sigma}_{1}\right)}\left[\mathfrak{\mathfrak { g }}_{2}^{\sigma_{1}}-\mathfrak{\mathfrak { g }}_{1}^{\sigma_{1}}\right]+\frac{\widehat{\mathfrak{u}}^{\star}}{r \Gamma\left(\hat{\sigma}_{1}-\hat{\sigma}_{3}\right)}\left[\mathfrak{\mathfrak { g }}_{2}^{\sigma_{1}-\dot{\sigma}_{3}}-\mathfrak{\mathfrak { g }}_{1}^{\hat{\sigma}_{1}-\hat{\sigma}_{3}}\right] \\
& +\Pi\left(\mathfrak{g}_{2}-\mathfrak{g}_{1}\right)\left[\left|a_{0}\right|+\dot{r}^{\star}{ }^{\star} r \Gamma\left(\hat{\sigma}_{1}-\dot{v}_{1}+1\right)\right. \\
& +\dot{r} \hat{\mathfrak{u}}^{\star} r \Gamma\left(\hat{\sigma}_{1}-\hat{\sigma}_{3}-\dot{v}_{1}+1\right)+(1-\hat{r}) \mathfrak{u}^{\star} r \Gamma\left(\hat{\sigma}_{1}-\dot{v}_{2}+1\right) \\
& \left.+(1-\hat{r}) \widehat{\mathfrak{u}}^{\star} r \dot{r} \Gamma\left(\hat{\sigma}_{1}-\hat{\sigma}_{3}-\dot{v}_{2}+1\right)\right] \text {. }
\end{aligned}
$$

The right-hand side of the above inequality is not dependent on and converges to 0 , as $\mathfrak{g}_{2}-\mathfrak{g}_{1} \longrightarrow 0$. This means that $P_{2}$ is equicontinuous and admits the relative compactness on $B_{\overline{\Delta_{2}}}$. Thus, Arzelà-Ascoli theorem ensures that $P_{2}$ is compact on $B_{\bar{\Delta}_{2}}$. Consequently, our $\mathbb{B V M P}(3)$ possesses at least one solution on $\mathcal{O}$. The proof is completed.

Example 2. Consider the following Liouville-Caputo $\mathbb{B V M P}$ :

$$
\left\{\begin{array}{l}
\left(\frac{2}{2 \mathfrak{C}}{ }^{C} \mathbb{D}_{0^{+}}^{15 / 8}+\frac{1}{2 \mathfrak{3}} \mathbb{0}^{2 / 7}\right) z(\mathfrak{I})=\frac{\sin ^{2} \mathfrak{g}}{(\pi+\mathfrak{g})^{2}}\left(\frac{|z(\mathfrak{g})|}{|z(\mathfrak{g})|+1}\right) \\
+{ }^{C} \mathbb{D}_{0^{+}}^{3 / 2}\left[\frac{1}{3 \exp (\mathfrak{g})+1}\left(\frac{|z(\mathfrak{g})|}{\pi^{2}+\exp (\mathfrak{g})|z(\mathfrak{g})|}\right)\right], \\
z(0)=0, \frac{2}{5} C \mathbb{D}_{0^{+}}^{1 / 8} z(1)+\frac{3}{5} C_{\mathbb{D}_{0^{+}}}^{1 / 4} z(1)=\frac{22}{7} .
\end{array}\right.
$$

Now, we have $\hat{\sigma}_{1}=15 / 8 \in(1,2] ., \quad \hat{\sigma}_{2}=2 / 7 \in(0,1]$, $\hat{\sigma}_{3}=3 / 2 \in(0,1], \quad \dot{r}=22 / 23 \in(0,1], \quad \dot{r}=2 / 5 \in(0,1]$, $\hat{v}_{1}=1 / 8 \in(0,1], \dot{v}_{2}=1 / 4 \in(0,1], a_{0}=22 / 7 \in \mathbb{R} \dot{v}_{1}, \tau^{*}=1$, and

$$
\begin{aligned}
\mathfrak{u}(\mathfrak{G}, z) & =\frac{\sin ^{2} \mathfrak{G}}{(\pi+\mathfrak{G})^{2}}\left(\frac{|z|}{|z|+1}\right), \quad \widehat{\mathfrak{u}}(\mathfrak{B}, z) \\
& =\frac{1}{3 \exp (\mathfrak{I})+1}\left(\frac{|z|}{\pi^{2}+\exp (\mathfrak{I})|z|}\right) .
\end{aligned}
$$

Hence, 


$$
\begin{aligned}
& \left|\mathfrak{u}(\mathfrak{G}, z)-\mathfrak{u}\left(\mathfrak{G}, z^{\star}\right)\right|=\left|\frac{\sin ^{2} \mathfrak{g}}{(\pi+\mathfrak{g})^{2}}\left(\frac{|z|}{|z|+1}\right)-\frac{\sin ^{2} \mathfrak{g}}{(\pi+\mathfrak{G})^{2}}\left(\frac{\left|z^{\star}\right|}{\left|z^{\star}\right|+1}\right)\right| \\
& =\frac{\sin ^{2} \mathfrak{g}}{(\pi+\mathfrak{g})^{2}}\left|\frac{|z|}{|z|+1}-\frac{\left|z^{\star}\right|}{\left|z^{\star}\right|+1}\right| \\
& \leq \frac{1}{\pi^{2}}\left|z-z^{\star}\right|, \\
& \left|\widehat{\mathfrak{u}}(\mathfrak{g}, z)-\widehat{\mathfrak{u}}\left(\mathfrak{g}, z^{\star}\right)\right|=\left|\frac{1}{3 \exp (\mathfrak{g})+1}\left(\frac{|z|}{\pi^{2}+\exp (\mathfrak{g})|z|}\right)-\frac{1}{3 \exp (\mathfrak{g})+1}\left(\frac{\left|z^{\star}\right|}{\pi^{2}+\exp (\mathfrak{g})\left|z^{\star}\right|}\right)\right| \\
& =\frac{1}{3 \exp (\mathfrak{g})+1}\left|\frac{|z|}{\pi^{2}+\exp (\mathfrak{g})|z|}-\frac{\left|z^{\star}\right|}{\pi^{2}+\exp (\mathfrak{g}) \mid z^{\star}}\right| \\
& \leq \frac{1}{4}\left|z-z^{\star}\right|,
\end{aligned}
$$

i.e., $\Theta_{1}=2 / \pi^{2}, \Theta_{2}=1 / 4$, and accordingly,

$\Pi=\left[\frac{\dot{r}}{\Gamma\left(2-\dot{v}_{1}\right)}+\frac{1-\dot{r}}{\Gamma\left(2-\dot{v}_{2}\right)}\right]^{-1}=\left[\frac{2 / 5}{\Gamma(2-1 / 8)}+\frac{1-2 / 5}{\Gamma(2-1 / 4)}\right]^{-1}$,

and

By some computations, we get $\Pi \approx 0.9325$, $\eta_{1} \approx 0.0415<1, \eta_{2} \approx 1.2287, \eta_{3} \approx 2.2275$, and

$$
\eta_{1}+\Theta_{1} \eta_{2}+\Theta_{2} \eta_{3} \approx 0.8473<1
$$

Also, we get

$$
\begin{aligned}
|\mathfrak{u}(\mathfrak{G}, z)| & =\left|\frac{\sin ^{2} \mathfrak{G}}{(\pi+\mathfrak{G})^{2}}\left(\frac{|z|}{|z|+1}\right)\right| \\
& =\left|\frac{\sin ^{2} \mathfrak{G}}{(\pi+\mathfrak{G})^{2}}\right| \frac{|z|}{|z|+1} \leq \frac{\sin ^{2} \mathfrak{G}}{(\pi+\mathfrak{G})^{2}}=\varrho_{1}(\mathfrak{I}), \\
|\widehat{\mathfrak{u}}(\mathfrak{G}, \mathrm{z})| & =\left|\frac{1}{3 \exp (\mathfrak{G})+1}\left(\frac{|z|}{\pi^{2}+\exp (\mathfrak{g})|z|}\right)\right| \\
& =\left|\frac{1}{3 \exp (\mathfrak{S})+1}\right|\left|\frac{|z|}{\pi^{2}+\exp (\mathfrak{I})|z|}\right| \leq \frac{1}{3 \exp (\mathfrak{I})+1}=\varrho_{2}(\mathfrak{I}) .
\end{aligned}
$$

Table 2 shows these results. These numerical data are plotted in Figure 2. Then, Theorem 2 states that the Liouville-Caputo $\mathbb{B V} \mathbb{P}(45)$ admits at least one solution on $\mathcal{O}$.

$$
\begin{aligned}
& \eta_{1}=\frac{1-\dot{r}}{r \Gamma\left(\dot{\sigma}_{1}+\dot{\sigma}_{2}+1\right)}+\frac{\dot{r}(1-\dot{r}) \Pi}{\dot{r} \Gamma\left(\dot{\sigma}_{1}+\dot{\sigma}_{2}-\dot{v}_{1}+1\right)}+\frac{(1-\dot{r})(1-\dot{r}) \Pi}{\dot{r} \Gamma\left(\dot{\sigma}_{1}+\dot{\sigma}_{2}-\dot{v}_{2}+1\right)} \\
& \eta_{2}=\frac{1}{r \Gamma\left(\dot{\sigma}_{1}+1\right)}+\frac{\dot{r} \Pi}{r \Gamma\left(\dot{\sigma}_{1}-\dot{v}_{1}+1\right)}+\frac{(1-\dot{r}) \Pi}{r \Gamma\left(\hat{\sigma}_{1}-\dot{v}_{2}+1\right)} \\
& =\frac{1-22 / 23}{22 / 23 \Gamma(15 / 8+2 / 7+1)}+\frac{2 / 5(1-22 / 23) \Pi}{22 / 23 \Gamma(15 / 8+2 / 7-1 / 4+1)}+\frac{(1-2 / 5)(1-22 / 23) \Pi}{22 / 23 \Gamma(15 / 8+2 / 7-1 / 4+1)}, \\
& =\frac{1}{22 / 23 \Gamma(15 / 8+1)}+\frac{2 / 5 \Pi}{22 / 23 \Gamma(15 / 8-1 / 8+1)}+\frac{(1-2 / 5) \Pi}{22 / 23 \Gamma(15 / 8-1 / 4+1)} \\
& \eta_{3}=\frac{1}{r \Gamma\left(\sigma_{1}-\dot{\sigma}_{3}+1\right)}+\frac{\dot{r} \Pi}{\dot{r} \Gamma\left(\hat{\sigma}_{1}-\dot{\sigma}_{3}-\dot{v}_{1}+1\right)}+\frac{(1-\dot{r}) \Pi}{\dot{r} \Gamma\left(\dot{\sigma}_{1}-\dot{\sigma}_{3}-\dot{v}_{2}+1\right)} \\
& =\frac{1}{22 / 23 \Gamma(15 / 8-3 / 2+1)}+\frac{2 / 5 \Pi}{22 / 23 \Gamma(15 / 8-3 / 2-1 / 8+1)}+\frac{(1-2 / 5) \Pi}{22 / 23 \Gamma(15 / 8-3 / 2-1 / 4+1)} .
\end{aligned}
$$


TABLe 2: Numerical values of $\eta_{1}, \eta_{2}, \eta_{3}$, and $\Pi$, for $r \in(0,1]$, in Example 2.

\begin{tabular}{|c|c|c|c|c|c|}
\hline \multirow[b]{2}{*}{$\dot{r}$} & \multicolumn{5}{|c|}{$\dot{r} \in(0,1]$} \\
\hline & $\Pi$ & $\eta_{1}$ & $\eta_{2}$ & $\eta_{3}$ & $\eta_{1}+\Theta_{1} \eta_{2}+\Theta_{2} \eta_{3}$ \\
\hline 0.04 & 0.9325 & 20.0781 & 27.0324 & 49.0041 & 37.8071 \\
\hline 0.09 & 0.9325 & 9.5828 & 13.5162 & 24.5020 & 18.4472 \\
\hline 0.13 & 0.9325 & 6.0843 & 9.0108 & 16.3347 & 11.9939 \\
\hline 0.17 & 0.9325 & 4.3351 & 6.7581 & 12.2510 & 8.7673 \\
\hline 0.22 & 0.9325 & 3.2855 & 5.4065 & 9.8008 & 6.8313 \\
\hline 0.26 & 0.9325 & 2.5858 & 4.5054 & 8.1673 & 5.5406 \\
\hline 0.30 & 0.9325 & 2.0860 & 3.8618 & 7.0006 & 4.6187 \\
\hline 0.35 & 0.9325 & 1.7112 & 3.3791 & 6.1255 & 3.9273 \\
\hline 0.39 & 0.9325 & 1.4197 & 3.0036 & 5.4449 & 3.3895 \\
\hline 0.43 & 0.9325 & 1.1864 & 2.7032 & 4.9004 & 2.9593 \\
\hline 0.48 & 0.9325 & 0.9956 & 2.4575 & 4.4549 & 2.6073 \\
\hline 0.52 & 0.9325 & 0.8366 & 2.2527 & 4.0837 & 2.3140 \\
\hline 0.57 & 0.9325 & 0.7020 & 2.0794 & 3.7695 & 2.0658 \\
\hline 0.61 & 0.9325 & 0.5867 & 1.9309 & 3.5003 & 1.8531 \\
\hline 0.65 & 0.9325 & 0.4867 & 1.8022 & 3.2669 & 1.6687 \\
\hline 0.70 & 0.9325 & 0.3993 & 1.6895 & 3.0628 & 1.5073 \\
\hline 0.74 & 0.9325 & 0.3221 & 1.5901 & 2.8826 & 1.3650 \\
\hline 0.78 & 0.9325 & 0.2535 & 1.5018 & 2.7224 & 1.2385 \\
\hline 0.83 & 0.9325 & 0.1921 & 1.4228 & 2.5792 & 1.1252 \\
\hline 0.87 & 0.9325 & 0.1369 & 1.3516 & 2.4502 & 1.0233 \\
\hline 0.91 & 0.9325 & 0.0869 & 1.2873 & 2.3335 & 0.9312 \\
\hline $22 / 23 \simeq 0.96$ & 0.9325 & 0.0415 & 1.2287 & 2.2275 & 0.8473 \\
\hline 1.00 & 0.9325 & 0.0000 & 1.1753 & 2.1306 & 0.7708 \\
\hline
\end{tabular}

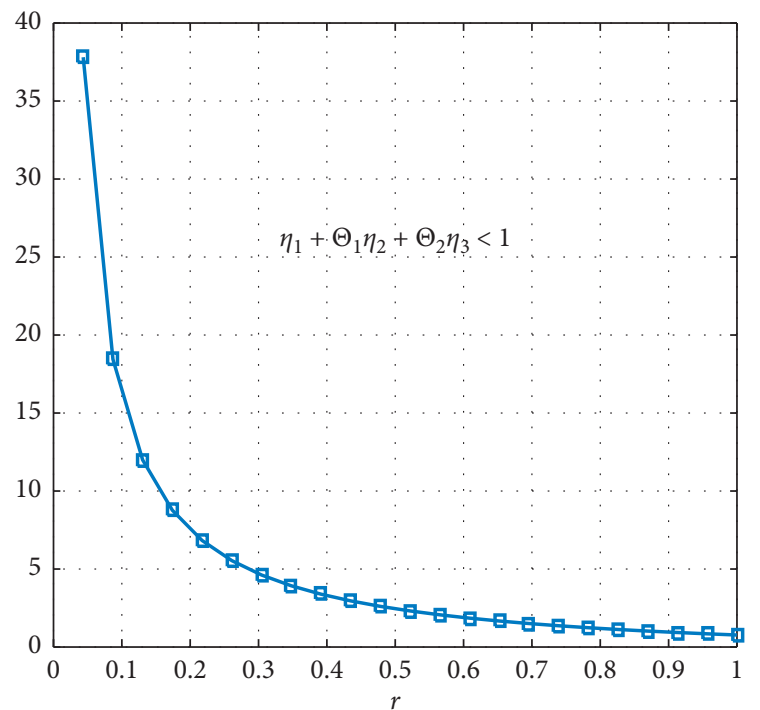

(a)

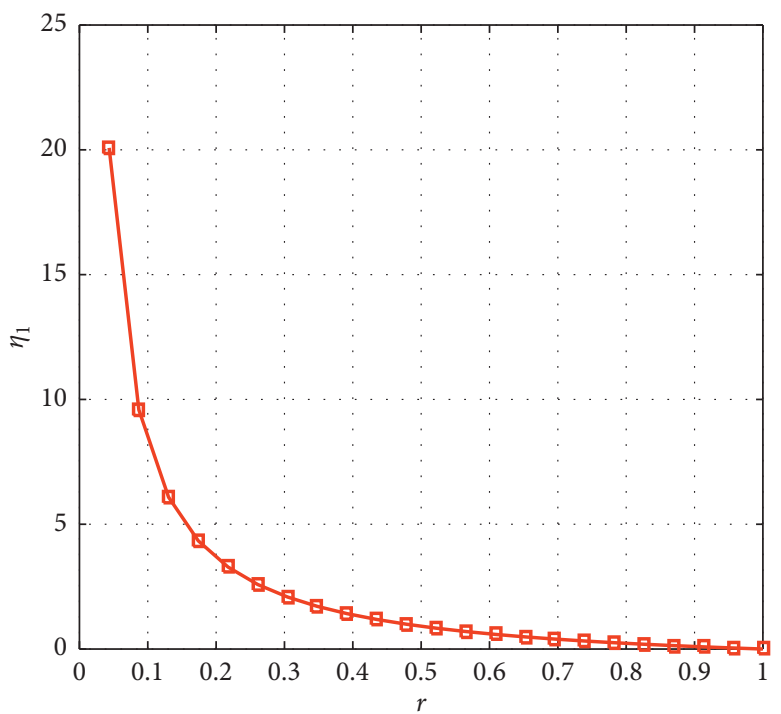

(b)

FiguRe 2: Graphical representation of $\eta_{1}+\Theta_{1} \eta_{2}+\Theta_{2} \eta_{3}$ and $\eta_{1}<1$ in Example 2. (a) $r \in(0,1], \dot{r}=2 / 5$. (b) $\eta_{1}$ vs $r=22 / 23, \dot{r}=2 / 5$. 
3.3. Existence Result by Using Nonlinear Alternative of Leray-Schauder. Another result of existence criterion is realized by implementing the hypotheses in Lemma 2. The desired criterion is proved below by the next theorem.

Theorem 3. Assume that $\mathfrak{u}, \widehat{\mathfrak{u}}: \mathcal{O} \times \mathbb{R} \longrightarrow \mathbb{R}$ are two continuous functions which satisfy the following assumption.

$\left(\mathcal{S}_{5}\right)$ : there are two continuous nondecreasing functions $\wp_{1}, \wp_{2}:[0,+\infty) \longrightarrow(0,+\infty)$ and two functions $\hbar_{1}, \hbar_{2} \in C(\mathcal{O}, \mathbb{R})$ provided that

$$
\begin{gathered}
|\mathfrak{u}(\mathfrak{G}, z)| \leq \hbar_{1}(\mathfrak{g}) \wp_{1}(\|z\|), \\
|\widehat{\mathfrak{u}}(\mathfrak{g}, z)| \leq \hbar_{2}(\mathfrak{g}) \wp_{2}(\|z\|),
\end{gathered}
$$

for all $(\mathfrak{S}, z) \in \mathcal{O} \times \mathbb{R} ;$ moreover, the following assumption holds.

$\left(\mathcal{S}_{6}\right)$ : there exists a positive real constant $\bar{\Delta}_{3}$ so that

$$
\frac{\left\|\hbar_{1}\right\| \wp_{1}\left(\bar{\Delta}_{3}\right) \eta_{2}+\left\|\hbar_{2}\right\| \wp_{2}\left(\bar{\Delta}_{3}\right) \eta_{3}+\Pi\left|a_{0}\right|}{\bar{\Delta}_{3}\left(1-\eta_{1}\right)}>1, \eta_{1}<1 .
$$

Then, the Liouville-Caputo $\mathbb{B M P}$ (3) has at least one solution on $\mathcal{O}$, where $\Pi, \eta_{1}, \eta_{2}, \eta_{3}$ stand for the same constants introduced, respectively, by expressions (11), (21), (22), and (23).

Proof. Consider again the operator $P$ expressed as (20). First, we will prove that $P$ maps bounded sets into bounded sets in $C(\mathcal{O}, \mathbb{R})$. Let

$$
\mathbb{B}_{\delta}=\{z \in C(\mathcal{O}, \mathbb{R}):\|z\| \leq \delta\}
$$

be a bounded set in $C(\mathcal{O}, \mathbb{R})$, where $\delta$ is a real positive number $(\delta>0)$. For each $\mathfrak{s} \in \mathcal{O}$, we have

$$
\begin{aligned}
& P z(\mathfrak{g}) \leq \frac{1-\dot{r}}{\dot{r} \Gamma\left(\hat{\sigma}_{1}+\dot{\sigma}_{2}\right)} \int_{0}^{1}(1-\xi)^{\hat{\sigma}_{1}+\dot{\sigma}_{2}-1}|z(\xi)| d \xi
\end{aligned}
$$

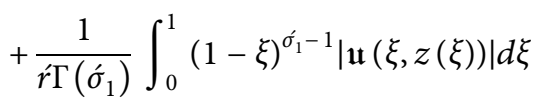

$$
\begin{aligned}
& +\frac{1}{r \Gamma\left(\dot{\sigma}_{1}-\sigma_{3}\right)} \int_{0}^{1}(1-\xi)^{\sigma_{1}-\dot{\sigma}_{3}-1}|\widehat{\mathfrak{u}}(\xi, z(\xi))| d \xi \\
& +\Pi\left[\left|a_{0}\right|+\frac{\dot{r}\left(1-\dot{r}^{\prime}\right)}{\dot{r} \Gamma\left(\dot{\sigma}_{1}+\dot{\sigma}_{2}-\dot{v}_{1}\right)} \int_{0}^{1}(1-\xi)^{\sigma_{1}+\dot{\sigma}_{2}-\dot{v}_{1}-1}|z(\xi)| d \xi\right. \\
& +\frac{\dot{r}}{r \Gamma\left(\dot{\sigma}_{1}-\dot{v}_{1}\right)} \int_{0}^{1}(1-\xi)^{\hat{\sigma}_{1}-\hat{\nu}_{1}-1}|\mathfrak{u}(\xi, z(\xi))| d \xi \\
& +\frac{\dot{r}}{\dot{r} \Gamma\left(\dot{\sigma}_{1}-\dot{\sigma}_{3}-\dot{v}_{1}\right)} \int_{0}^{1}(1-\xi)^{\sigma_{1}-\dot{\sigma}_{3}-\dot{\nu}_{1}-1}|\widehat{\mathfrak{u}}(\xi, z(\xi))| d \xi \\
& +\frac{\left(1-\dot{r}^{\prime}\right)\left(1-\dot{r}^{\prime}\right)}{r \Gamma\left(\hat{\sigma}_{1}+\hat{\sigma}_{2}-\dot{v}_{2}\right)} \int_{0}^{1}(1-\xi)^{\hat{\sigma}_{1}+\dot{\sigma}_{2}-\hat{v}_{2}-1}|z(\xi)| d \xi
\end{aligned}
$$

$$
\begin{aligned}
& +\frac{1-\dot{r}}{r \Gamma\left(\sigma_{1}-\dot{v}_{2}\right)} \int_{0}^{1}(1-\xi)^{\sigma_{1}-\dot{v}_{2}-1}|\mathfrak{u}(\xi, z(\xi))| d \xi \\
& \left.+\frac{1-\dot{r}}{\dot{r} \Gamma\left(\hat{\sigma}_{1}-\dot{\sigma}_{3}-\dot{v}_{2}\right)} \int_{0}^{1}(1-\xi)^{\sigma_{1}-\dot{\sigma}_{3}-\dot{v}_{2}-1}|\mathfrak{u}(\xi, z(\xi))| d \xi\right] \\
& \leq\|z\| \eta_{1}+\left\|\hbar_{1}\right\| \wp_{1}(z) \eta_{2}+\left\|\hbar_{2}\right\| \wp_{2}(\|z\|) \eta_{3}+\Pi\left|a_{0}\right| \\
& \leq \delta \eta_{1}+\left\|\hbar_{1}\right\| \wp_{1}(\delta) \eta_{2}+\left\|\hbar_{2}\right\| \wp_{2}(\delta) \eta_{3}+\Pi\left|a_{0}\right|
\end{aligned}
$$

and consequently,

$$
\|P z\| \leq \delta \eta_{1}+\left\|\hbar_{1}\right\| \wp_{1}(\delta) \eta_{2}+\left\|\hbar_{2}\right\| \wp_{2}(\delta) \eta_{3}+\Pi\left\|a_{0}\right\| .
$$

The next property is that we prove that the operator $P$ maps the bounded sets to the equicontinuous sets. Let $\mathfrak{u}^{\star}=\sup _{(\mathfrak{g}, z) \in \mathcal{O} \times B_{\delta}}|\mathfrak{u}(\mathfrak{g}, z)|$, and $\widehat{\mathfrak{u}}^{\star}=\sup _{(\mathfrak{g}, z) \in \mathcal{O} \times B_{\delta}}|\widehat{\mathfrak{u}}(\mathfrak{g}, z)|$.

So, for $\mathfrak{g}_{1}, \mathfrak{g}_{2} \in \mathcal{O}$ with $\mathfrak{g}_{1}<\mathfrak{g}_{2}$ and $\mathrm{z} \in B_{\delta}$, we have

$$
\begin{aligned}
& \left|P z\left(\mathfrak{g}_{2}\right)-P z\left(\mathfrak{g}_{1}\right)\right| \leq \frac{\delta(1-\dot{r})}{r \Gamma\left(\hat{\sigma}_{1}+\dot{\sigma}_{2}\right)} \\
& \cdot\left[\int_{0}^{\mathfrak{S}_{1}}\left[\left(\mathfrak{S}_{2}-\xi\right)^{\hat{\sigma}_{1}+\dot{\sigma}_{2}-1}-\left(\mathfrak{S}_{1}-\xi\right)^{\hat{\sigma}_{1}+\tilde{\sigma}_{2}-1}\right] d \xi\right. \\
& \left.+\int_{\mathfrak{S}_{1}}^{\mathfrak{g}_{2}}\left(\mathfrak{I}_{2}-\xi\right)^{\sigma_{1}+\dot{\sigma}_{2}-1} d \xi\right] \\
& +\frac{\mathfrak{u}^{\star}}{r \Gamma\left(\hat{\sigma}_{1}\right)}\left[\int_{0}^{\mathfrak{s}_{1}}\left[\left(\mathfrak{g}_{2}-\xi\right)^{\hat{\sigma}_{1}-1}-\left(\mathfrak{g}_{1}-\xi\right)^{\hat{\sigma}_{1}-1}\right] d \xi\right. \\
& \left.+\int_{\mathfrak{S}_{1}}^{\mathfrak{g}_{2}}\left(\mathfrak{g}_{2}-\xi\right)^{\sigma_{1}-1} d \xi\right] \\
& +\frac{\widehat{\mathfrak{t}}^{\star}}{r \Gamma\left(\hat{\sigma}_{1}-\sigma_{3}\right)} \\
& \cdot\left[\int_{0}^{\mathfrak{g}_{1}}\left[\left(\mathfrak{g}_{2}-\xi\right)^{\hat{\sigma}_{1}-\hat{\sigma}_{3}-1}-\left(\mathbf{t}_{1}-\xi\right)^{\hat{\sigma}_{1}-\hat{\sigma}_{3}-1}\right] d \xi\right. \\
& \left.+\int_{\mathfrak{g}_{1}}^{\mathfrak{g}_{2}}\left(\mathfrak{g}_{2}-\xi\right)^{\dot{\sigma}_{1}-\dot{\sigma}_{3}-1} d \xi\right]+\Pi\left(\mathfrak{I}_{2}-\mathfrak{g}_{1}\right)\left[\left|a_{0}\right|\right. \\
& +\frac{\dot{r}(1-\dot{r}) \delta}{\dot{r} \Gamma\left(\dot{\sigma}_{1}+\dot{\sigma}_{2}-\dot{v}_{1}\right)} \int_{0}^{1}(1-\xi)^{\dot{\sigma}_{1}+\dot{\sigma}_{2}-\dot{v}_{1}^{\prime}-1} d \xi \\
& +\frac{\dot{r}_{\mathfrak{u}}{ }^{\star}}{r \Gamma\left(\dot{\sigma}_{1}-\dot{v}_{1}\right)} \int_{0}^{1}(1-\xi)^{\sigma_{1}-\hat{\nu}_{1}^{\prime}-1} d \xi \\
& +\frac{\hat{r} \hat{\mathfrak{u}}^{\star}}{r \Gamma\left(\hat{\sigma}_{1}-\hat{\sigma}_{3}-\dot{v}_{1}\right)} \int_{0}^{1}(1-\xi)^{\hat{\sigma}_{1}-\hat{\sigma}_{3}-\hat{v}_{1}-1} d \xi \\
& +\frac{\left(1-\dot{r}^{\prime}\right)(1-\dot{r}) \delta}{\dot{r} \Gamma\left(\hat{\sigma}_{1}-\hat{\sigma}_{3}-\dot{v}_{2}\right)} \int_{0}^{1}(1-\xi)^{\hat{\sigma}_{1}-\sigma_{3}-\hat{v}_{2}-1} d \xi
\end{aligned}
$$




$$
\begin{aligned}
& +\frac{\left(1-\dot{r}^{\prime}\right) \mathfrak{u t}^{\star}}{r \Gamma\left(\hat{\sigma}_{1}-\dot{v}_{2}\right)} \int_{0}^{1}(1-\xi)^{\sigma_{1}^{\prime}-\hat{v}_{2}^{\prime}-1} d \xi \\
& \left.+\frac{(1-\hat{r}) \widehat{\mathbf{u}}^{\star}}{r \Gamma\left(\hat{\sigma}_{1}-\hat{\sigma}_{3}-\dot{v}_{2}\right)} \int_{0}^{1}(1-\xi)^{\sigma_{1}^{\prime}-\dot{\sigma}_{3}-\hat{v}_{2}-1} d \xi\right] \\
& \leq \delta\left[\frac{(1-\hat{r})\left(\mathfrak{g}_{2}^{\sigma_{1}+\dot{\sigma}_{2}}-\mathfrak{g}_{1}^{\sigma_{1}+\dot{\sigma}_{2}}\right)}{\dot{r} \Gamma\left(\hat{\sigma}_{1}+\dot{\sigma}_{2}+1\right)}+\frac{\Pi \dot{r}(1-\dot{r})\left(\mathfrak{g}_{2}-\mathfrak{g}_{1}\right)}{\dot{r} \Gamma\left(\hat{\sigma}_{1}+\hat{\sigma}_{2}-\hat{\nu}_{1}+1\right)}\right. \\
& \left.+\frac{\Pi(1-\dot{r})(1-\hat{r})\left(\mathfrak{g}_{2}-\mathfrak{g}_{1}\right)}{\dot{r} \Gamma\left(\hat{\sigma}_{1}-\hat{\sigma}_{3}-\dot{v}_{2}+1\right)}\right] \\
& +\mathfrak{u}^{\star}\left[\frac{\left(\mathfrak{g}_{2}^{\sigma_{1}}-\mathfrak{g}_{1}^{\sigma_{1}}\right)}{\hat{r} \Gamma\left(\hat{\sigma}_{1}+1\right)}+\frac{\Pi \dot{r}\left(\mathfrak{g}_{2}-\mathfrak{g}_{1}\right)}{r \Gamma\left(\hat{\sigma}_{1}-\dot{v}_{1}+1\right)}+\frac{\Pi(1-\dot{r})\left(\mathfrak{g}_{2}-\mathfrak{g}_{1}\right)}{r \Gamma\left(\hat{\sigma}_{1}-\dot{v}_{2}+1\right)}\right] \\
& +\widehat{\mathfrak{u}}^{\star}\left[\frac{\left(\mathfrak{g}_{2}^{\hat{\sigma}_{1}-\hat{\sigma}_{3}^{\prime}}-\mathfrak{g}_{1}^{\hat{\sigma}_{1}-\hat{\sigma}_{3}}\right)}{r \Gamma\left(\hat{\sigma}_{1}-\hat{\sigma}_{3}+1\right)}+\frac{\Pi \dot{r}\left(\mathfrak{g}_{2}-\mathfrak{g}_{1}\right)}{r \Gamma\left(\hat{\sigma}_{1}-\hat{\sigma}_{3}-\dot{\nu}_{1}+1\right)}\right. \\
& \left.+\frac{\Pi(1-\hat{r})\left(\mathfrak{g}_{2}-\mathfrak{g}_{1}\right)}{r \Gamma\left(\hat{\sigma}_{1}-\hat{\sigma}_{3}-\hat{v}_{2}+1\right)}\right]+\Pi\left|a_{0}\right|\left(\mathfrak{g}_{2}-\mathfrak{g}_{1}\right) \text {. }
\end{aligned}
$$

Note that the second side of the last inequality is not dependent on $\mathrm{z}$ and goes to 0 when $\mathfrak{g}_{2}-\mathfrak{g}_{1} \longrightarrow 0$. Hence, by Arzelà-Ascoli theorem, it is figured out that $P$ is completely continuous. At this moment, we have established the boundedness of the set of solutions for the operator equation $\mathrm{z}=\ell P \mathrm{z}$, where $\ell \in \mathcal{O}$. Let now $\mathrm{z}$ be a solution of $\mathbb{B V} \mathbb{P}(3)$. With the same arguments used in (56), one can find

$$
\|z\| \leq\|z\| \eta_{1}+\left\|\hbar_{1}\right\| \wp_{1}(\|z\|) \eta_{2}+\left\|\hbar_{2}\right\| \wp_{2}(\|z\|) \eta_{3}+\Pi\left|a_{0}\right|,
$$

and we can also write it as

$$
\frac{\|z\|\left(1-\eta_{1}\right)}{\left\|\hbar_{1}\right\| \wp_{1}(\|z\|) \eta_{2}+\left\|\hbar_{2}\right\| \wp_{2}(\|z\|) \eta_{3}+\Pi\left|a_{0}\right|} \leq 1 .
$$

From assumption $\left(\mathcal{S}_{6}\right)$, there exists a constant $\bar{\Delta}_{3}>0$ so that $\bar{\Delta}_{3} \neq\|z\|$. Consider the set

$$
\mathrm{E}=\left\{z \in C(\mathcal{O}, \mathbb{R}):\|z\|<\bar{\Delta}_{3}\right\} .
$$

It was proved that $P: \overline{\mathscr{E}} \longrightarrow C(\mathcal{O}, \mathbb{R})$ is a continuous and completely continuous operator. The selection of the set $\mathscr{E}$ allows us to confirm that there is no $z \in \partial \mathrm{E}$ which satisfies $z=\ell P z$, for $\ell \in \mathcal{O}$. Hence, the requirements of Lemma 2 ensure that $\mathscr{E}$ involves a fixed point $z^{*} \in \overline{\mathrm{E}}$ which stands for the solution to our Liouville-Caputo $\mathbb{B} \mathbb{M P}(3)$, and the proof is completed.

Example 3. Consider the Liouville-Caputo $\mathbb{B V} \mathbb{P}$

$$
\left\{\begin{array}{l}
\left(\frac{17}{19}{ }^{C} \mathbb{D}_{0^{+}}^{19 / 2}+\frac{2}{19} \square^{5 / 12}\right) z(\mathfrak{g})=\frac{2}{101(\mathfrak{g}+1)}\left(\frac{\|z(\mathfrak{g})\|^{2}}{\|z(\mathfrak{g})\|+1}+1\right) \\
+{ }^{C} \mathbb{D}_{0^{+}}^{17 / 12}\left[\frac{1}{10(3 \exp (\mathfrak{g})+1)}\left(\frac{\|z(\mathfrak{g})\|^{3}}{z^{2}(\mathfrak{g})+1}+\frac{5 z^{2}(\mathfrak{g})}{z^{2}(\mathfrak{I})+3}\right)\right], \\
\mathfrak{u}(0)=0, \frac{5}{11}{ }^{C} \mathbb{D}_{0^{+}}^{1 / 8} z(1)+\frac{6}{11} C_{\mathbb{D}_{0^{+}}}^{1 / 7} z(1)=\frac{4}{7} .
\end{array}\right.
$$

Now, we have $\hat{\sigma}_{1}=19 / 12 \in(1,2], \hat{\sigma}_{2}=5 / 12 \in(0,1]$, $\hat{\sigma}_{3}=17 / 12 \in(1,2], \quad \dot{r}=17 / 19 \in(0,1], \quad \dot{r}=5 / 1 \in(0,1]$, $\tau^{*}=1, \dot{v}_{1}=1 / 8 \in(0,1], \hat{v}_{2}=1 / 7 \in(0,1], a_{0}=4 / 7$, and

$$
\begin{aligned}
& \mathfrak{u}(\mathfrak{G}, z)=\frac{2}{101(\mathfrak{I}+1)}\left(\frac{|z(\mathfrak{G})|^{2}}{|z(\mathfrak{g})|+1}+1\right), \\
& \widehat{\mathfrak{u}}(\mathfrak{G}, z)=\frac{1}{10(3 \exp (\mathfrak{g})+1)}\left(\frac{|z(\mathfrak{G})|^{3}}{z^{2}(\mathfrak{I})+1}+\frac{5 z^{2}(\mathfrak{I})}{z^{2}(\mathfrak{I})+3}\right),
\end{aligned}
$$

and

$$
\begin{aligned}
|\mathfrak{u}(\mathfrak{g}, z)| & \leq \frac{2}{101(\mathfrak{B}+1)}(\|z\|+1), \\
|\widehat{z}(\mathfrak{g}, z)| & \leq \frac{1}{10(3 \exp (\mathfrak{I})+1)}(\|z\|+5) .
\end{aligned}
$$

Then,

$$
\begin{aligned}
& \hbar_{1}(\mathfrak{g})=\frac{2}{101(\mathfrak{B}+1)}, \wp_{1}(\|z\|)=\|z\|+1, \\
& \hbar_{2}(\mathfrak{\mathfrak { I }})=\frac{1}{10(3 \exp (\mathfrak{g})+1)}, \wp_{2}(\|z\|)=\|z\|+5 .
\end{aligned}
$$

On the other hand

$$
\Pi=\left[\frac{\dot{r}}{\Gamma\left(2-\dot{v}_{1}\right)}+\frac{1-\dot{r}}{\Gamma\left(2-\dot{v}_{2}\right)}\right]^{-1}=\left[\frac{5 / 11}{\Gamma(2-1 / 8)}+\frac{1-5 / 11}{\Gamma(2-1 / 7)}\right]^{-1},
$$

and 
TABLe 3: Numerical values of $\eta_{1}, \eta_{2}, \eta_{3}$, and $\Pi$, for and $\dot{r}=5 / 11$, in Example 3.

\begin{tabular}{|c|c|c|c|c|}
\hline$\dot{r}$ & $\Pi$ & $\eta_{1}$ & $\eta_{2}$ & $\eta_{3}$ \\
\hline 0.05 & 0.9504 & 18.6505 & 27.5308 & 38.8569 \\
\hline 0.11 & 0.9504 & 8.8072 & 13.7654 & 19.4284 \\
\hline 0.16 & 0.9504 & 5.5261 & 9.1769 & 12.9523 \\
\hline 0.21 & 0.9504 & 3.8855 & 6.8827 & 9.7142 \\
\hline 0.26 & 0.9504 & 2.9012 & 5.5062 & 7.7714 \\
\hline 0.32 & 0.9504 & 2.2450 & 4.5885 & 6.4761 \\
\hline 0.37 & 0.9504 & 1.7762 & 3.9330 & 5.5510 \\
\hline 0.42 & 0.9504 & 1.4247 & 3.4413 & 4.8571 \\
\hline 0.47 & 0.9504 & 1.1513 & 3.0590 & 4.3174 \\
\hline 0.53 & 0.9504 & 0.9325 & 2.7531 & 3.8857 \\
\hline 0.58 & 0.9504 & 0.7536 & 2.5028 & 3.5324 \\
\hline 0.63 & 0.9504 & 0.6044 & 2.2942 & 3.2381 \\
\hline 0.68 & 0.9504 & 0.4782 & 2.1178 & 2.9890 \\
\hline 0.74 & 0.9504 & 0.3700 & 1.9665 & 2.7755 \\
\hline 0.79 & 0.9504 & 0.2763 & 1.8354 & 2.5905 \\
\hline 0.84 & 0.9504 & 0.1943 & 1.7207 & 2.4286 \\
\hline $17 / 19 \simeq 0.89$ & 0.9504 & 0.1219 & 1.6195 & 2.2857 \\
\hline 0.95 & 0.9504 & 0.0576 & 1.5295 & 2.1587 \\
\hline 1.00 & 0.9504 & 0.0000 & 1.4490 & 2.0451 \\
\hline
\end{tabular}

$$
\begin{aligned}
& \eta_{1}=\frac{1-\dot{r}}{r \Gamma\left(\dot{\sigma}_{1}+\dot{\sigma}_{2}+1\right)}+\frac{\dot{r}(1-\dot{r}) \Pi}{\dot{r} \Gamma\left(\dot{\sigma}_{1}+\dot{\sigma}_{2}-\dot{v}_{1}+1\right)}+\frac{(1-\dot{r})(1-\dot{r}) \Pi}{\dot{r} \Gamma\left(\dot{\sigma}_{1}+\dot{\sigma}_{2}-\dot{v}_{2}+1\right)} \\
& =\frac{1-17 / 19}{17 / 19 \Gamma(19 / 12+5 / 12+1)}+\frac{5 / 11(1-17 / 19) \Pi}{17 / 19 \Gamma(19 / 12+5 / 12-1 / 8+1)}+\left(1-\frac{5}{11}\right)(1-17 / 19) \Pi / 17 / 19 \Gamma(19 / 12+5 / 12-1 / 7+1), \\
& \eta_{2}=\frac{1}{r \Gamma\left(\dot{\sigma}_{1}+1\right)}+\frac{r \Pi}{r \Gamma\left(\dot{\sigma}_{1}-\dot{v}_{1}+1\right)}+\frac{(1-\dot{r}) \Pi}{r \Gamma\left(\dot{\sigma}_{1}-\dot{v}_{2}+1\right)} \\
& =\frac{1}{17 / 19 \Gamma(19 / 12+1)}+\frac{5 / 11 \Pi}{17 / 19 \Gamma(19 / 12-1 / 8+1)}+\frac{(1-5 / 11) \Pi}{17 / 19 \Gamma(19 / 12-1 / 7+1)},
\end{aligned}
$$

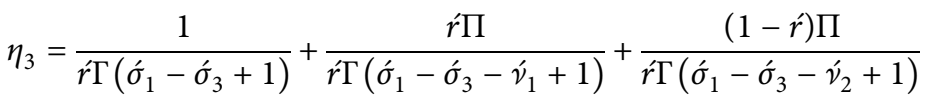

$$
\begin{aligned}
& =\frac{1}{17 / 19 \Gamma(19 / 12-17 / 12+1)}+5 / 11 \Pi / 17 / 19 \Gamma\left(19 / 12-17 / 12-\frac{1}{8}+1\right)+(1-5 / 11) \Pi / 17 / 19 \Gamma\left(19 / 12-17 / 12-\frac{1}{7}+1\right) \text {. }
\end{aligned}
$$

A simple computation leads to $\Pi \approx 0.9504, \eta_{1} \approx 0.1219$, $\eta_{2} \approx 1.6195$, and $\eta_{3} \approx 2.2857$. By solving the inequality (Table 3)

$$
\begin{aligned}
A & =\frac{\left\|\hbar_{1}\right\| \wp_{1}\left(\bar{\Delta}_{3}\right) \eta_{2}+\left\|\hbar_{2}\right\| \wp_{2}\left(\bar{\Delta}_{3}\right) \eta_{3}+\Pi\left|a_{0}\right|}{\bar{\Delta}_{3}\left(1-\eta_{1}\right)} \\
& =\frac{2 \times 1.6195 / 101\left(\bar{\Delta}_{3}+1\right)+2.2857 / 40\left(\bar{\Delta}_{3}+5\right)+0.9504 \times 4 / 7}{(1-0.1219) \bar{\Delta}_{3}}>1,
\end{aligned}
$$

we get $A>1.1000>1$. Table 4 shows these data. These numerical values are plotted in Figure 3.
Then, the assumption (6) holds for any $\bar{\Delta}_{3}>0.8456$. Consequently, from Theorem 3, we conclude that for 
TABLE 4: Numerical results of $\bar{\Delta}_{3}$ and $A$ based on Table 3 for $\eta_{1}, \eta_{2}$, $\eta_{3}$, and $\Pi$ in Example 3.

\begin{tabular}{lc}
\hline & $\Pi=0.9504, \eta_{1}=0.1219, \eta_{2}=1.6195, \eta_{3}=2.2857$ \\
\hline $\bar{\Delta}_{3}$ & $A>1$ \\
0.52 & 0.5357 \\
0.56 & 0.5720 \\
0.60 & 0.6076 \\
0.64 & 0.6426 \\
0.68 & 0.6770 \\
0.72 & 0.7109 \\
0.76 & 0.7442 \\
0.80 & 0.7769 \\
0.84 & 0.8091 \\
0.88 & 0.8408 \\
0.92 & 0.8720 \\
0.96 & 0.9027 \\
1.00 & 0.9329 \\
1.04 & 0.9626 \\
1.08 & 0.9919 \\
1.12 & $1.0207>1$ \\
1.16 & 1.0491 \\
1.20 & 1.0770 \\
1.24 & 1.1046 \\
1.28 & 1.1317 \\
1.32 & 1.1584 \\
\hline
\end{tabular}

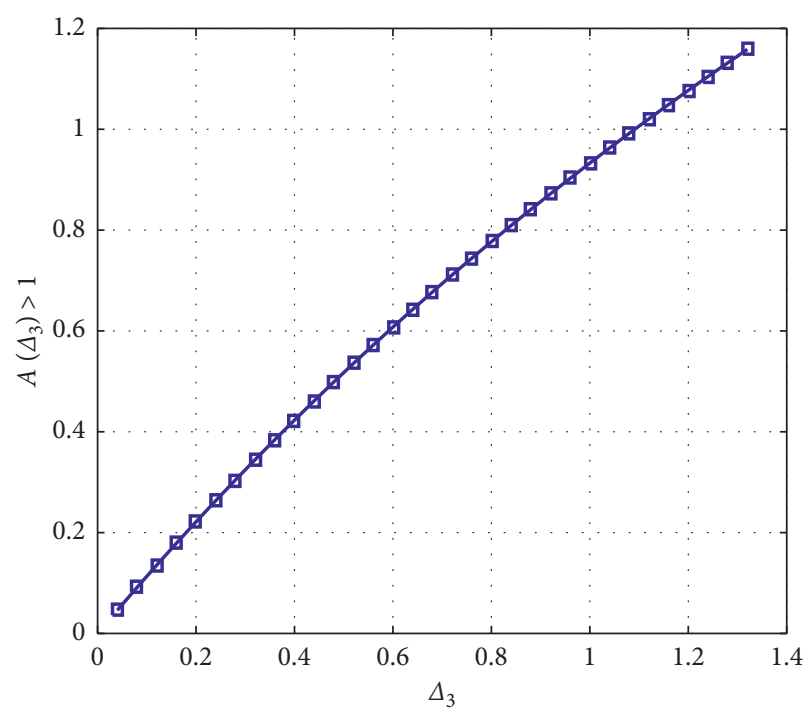

FIgURE 3: Graphical representation of $A>1$ for $\mathfrak{g} \in(0,1)$ vs. $\bar{\Delta}_{3}$

Liouville-Caputo $\mathbb{B M P}(62)$, at least one solution is found on 0.

\section{Conclusion}

In this paper, we considered a Liouville-Caputo $\mathbb{B V P}$ and proved our main results by using three fixed point theorems due to Banach, Krasnoselskii, and Leray-Schauder. Several special cases can be extracted from the mentioned $\mathbb{B V P}$ (3). Let us point out them, for example, if $\dot{r}=1$, then the Liouville-Caputo $\mathbb{B V} \mathbb{P}(3)$ reduces to the following one:

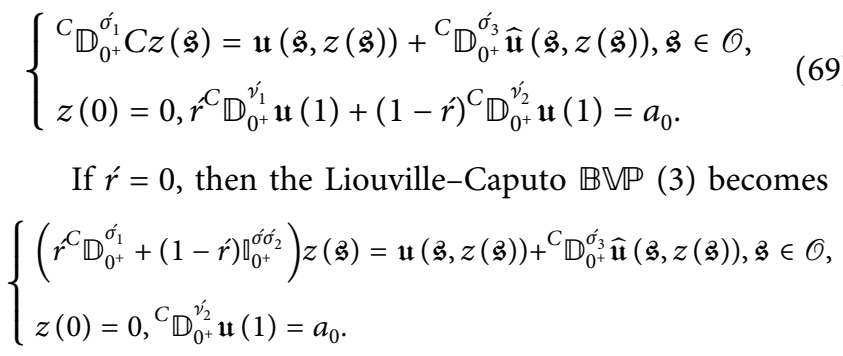

Consequently, some existence and uniqueness results for this particular case are obtained by exploiting Theorems 1-3. For future studies, we aim to combine these $\mathbb{B V P}$ s with nonsingular kernels in fractal-fractional operators.

\section{Data Availability}

No data were used to support this study.

\section{Conflicts of Interest}

The authors declare that they have no conflicts of interest.

\section{Authors' Contributions}

This study was realized in collaboration with equal responsibility. All authors read and approved the final manuscript.

\section{Acknowledgments}

The fourth author would like to thank Bu-Ali Sina University, and the first and fifth authors would like to thank Azarbaijan Shahid Madani University.

\section{References}

[1] A. A. Kilbas, H. M. Srivastava, and J. J. Trujillo, Theory and Applications of Fractional Differential Equations, Elsevier, Amsterdam, Netherlands, 2006.

[2] K. S. Miller and B. Ross, An Introduction to Fractional Calculus and Fractional Differential Equations, Wiley, New York, NY, USA, 1993.

[3] I. Podlubny, Fractional Differential Equations, Academic Press, San Diego, CA, USA, 1999.

[4] B. Ahmad and R. P. Agarwal, "Some new versions of fractional boundary value problems with slit-strips conditions," Boundary Value Problems, vol. 175, 2014.

[5] B. Ahmad and S. K. Ntouyas, "Existence results for Caputo type sequential fractional differential inclusions with nonlocal integral boundary conditions," Journal of Applied Mathematics and Computing, vol. 50, no. 1-2, pp. 157-174, 2016.

[6] A. Alsaedi, S. K. Ntouyas, R. P. Agarwal, and B. Ahmad, "On Caputo type sequential fractional differential equations with nonlocal integral boundary conditions," Advances in Difference Equations, vol. 33, 2015.

[7] D. Boucenna, A. Boulfoul, A. Chidouh, A. Ben Makhlouf, and B. Tellab, "Some results for initial value problem of nonlinear fractional equation in Sobolev space," Journal of Applied Mathematics and Computing, vol. 67, no. 1-2, pp. 605-621, 2021. 
[8] B. Azzaoui, B. Tellab, and K. Zennir, Positive Solutions for Integral Nonlinear Boundary Value Problem in Fractional Sobolev Space, Wiley, Hoboken, NJ, USA, 2021.

[9] Z. Bai and W. Sun, "Existence and multiplicity of positive solutions for singular fractional boundary value problems," Computers \& Mathematics with Applications, vol. 63, no. 9, pp. 1369-1381, 2012.

[10] Z. Islam, M. Sarwar, and M. de la Sen, "Fixed-point results for generalized $\alpha$-admissible Hardy-Rogers' contractions in cone $b_{2}$-metric spaces over Banach's algebras with application," Advances in Mathematical Physics, vol. 2020, p. 8826060, 2020.

[11] M. Shoaib, M. Sarwar, and P. Kumam, "Multi-valued fixed point theorem via F- contraction of Nadler type and application to functional and integral equations," Boletim da Sociedade Paranaense de Matemática, vol. 39, no. 4, pp. 83-95, 2021.

[12] A. Ali, M. Sarwar, M. B. Zada, and K. Shah, "Existence of solution to fractional differential equation with fractional integral type boundary conditions," Mathematical Methods in the Applied Sciences, vol. 44, no. 2, pp. 1615-1627, 2021.

[13] A. Boulfoul, B. Tellab, N. Abdellouahab, and K. Zennir, "Existence and uniqueness results for initial value problem of nonlinear fractional integro-differential equation on an unbounded domain in a weighted Banach space," Mathematical Methods in the Applied Sciences, vol. 44, no. 5, pp. 3509-3520, 2021.

[14] W. Shatanawi, A. Boutiara, M. S. Abdo, M. B. Jeelani, and K. Abodayeh, "Nonlocal and multiple-point fractional boundary value problem in the frame of a generalized Hilfer derivative," Advances in Difference Equations, vol. 2021, no. 1, p. 294, 2021.

[15] A. Zada, J. Alzabut, H. Waheed, and I.-L. Popa, "Ulam-Hyers stability of impulsive integrodifferential equations with Riemann-Liouville boundary conditions," Advances in Difference Equations, vol. 2020, no. 1, p. 64, 2020.

[16] S. Sitho, S. Etemad, B. Tellab, S. Rezapour, S. K. Ntouyas, and J. Tariboon, "Approximate solutions of an extended multiorder boundary value problem by implementing two numerical algorithms," Symmetry, vol. 13, no. 8, p. 1341, 2021.

[17] S. Rezapour, S. Etemad, B. Tellab, P. Agarwal, and J. L. Garcia Guirao, "Numerical solutions caused by DGJIM and ADM methods for multi-term fractional BVP involving the generalized $\psi$-RL-operators," Symmetry, vol. 13 , no. 4, p. 532, 2021.

[18] H. Khan, F. Jarad, T. Abdeljawad, and A. Khan, "A singular ABC-fractional differential equation with p-Laplacian operator," Chaos, Solitons \& Fractals, vol. 129, pp. 56-61, 2019.

[19] H. Hasib Khan, C. Tunc, W. Cemil Tunc, A. Wen Chen, and fnm Aziz Khan, "Existence theorems and hyers-ulam stability for a class of hybrid fractional differential equations with $\mathrm{P}$ -laplacian operator," Journal of Applied Analysis \& Computation, vol. 8, no. 4, pp. 1211-1226, 2018.

[20] R. Rizwan, A. Zada, M. Ahmad, S. O. Shah, and H. Waheed, "Existence theory and stability analysis of switched coupled system of nonlinear implicit impulsive Langevin equations with mixed derivatives," Mathematical Methods in the Applied Sciences, vol. 44, no. 11, pp. 8963-8985, 2021.

[21] S. Etemad, S. Rezapour, and M. Esmael Samei, "On a fractional Caputo-Hadamard inclusion problem with sum boundary value conditions by using approximate endpoint property," Mathematical Methods in the Applied Sciences, vol. 43, no. 17, pp. 9719-9734, 2020.
[22] M. E. Samei, V. Hedayati, and Sh. Rezapour, "Existence results for a fraction hybrid differential inclusion with CaputoHadamard type fractional derivative," Advances in Difference Equations, vol. 163, 2019.

[23] M. Sher, K. Shah, M. Fečkan, and R. A. Khan, "Qualitative analysis of multi-terms fractional order delay differential equations via the topological degree theory," Mathematics, vol. 8 , no. 2, p. $218,2020$.

[24] T. Abdeljawad, R. P. Agarwal, E. Karapınar, and P. S. Kumari, "Solutions of the nonlinear integral equation and fractional differential equation using the technique of a fixed point with a numerical experiment in extended b-metric space," Symmetry, vol. 11, no. 5, p. 686, 2019.

[25] A. Boutiara, M. Benbachir, and K. Guerbati, "Caputo type fractional differential equation with nonlocal Erdélyi-Kober type integral boundary conditions in Banach spaces," Surveys in Mathematics and its Applications, vol. 15, pp. 399-418, 2020.

[26] S. Etemad, S. Rezapour, and M. E. Samei, “ $\alpha-\psi$-contractions and solutions of a q-fractional differential inclusion with three-point boundary value conditions via computational results," Advances in Difference Equations, vol. 2020, no. 1, p. $218,2020$.

[27] T. Sitthiwirattham, "On nonlocal fractional $q$-integral boundary value problems of fractional $q$-difference and fractional $q$-integro-difference equations involving different numbers of order and q," Boundary Value Problems, vol. 2016, p. 12, 2016.

[28] S. Sitho, C. Sudprasert, S. K. Ntouyas, and J. Tariboon, "Noninstantaneous impulsive fractional quantum Hahn integro-difference boundary value problems," Mathematics, vol. 8 , no. 5 , p. $671,2020$.

[29] M. E. Samei, R. Ghaffari, S.-W. Yao, M. K. A. Kaabar, F. Martínez, and M. Inc, "Existence of solutions for a singular fractional q-differential equations under riemann-liouville integral boundary condition," Symmetry, vol. 13, no. 7 , p. $1235,2021$.

[30] R. M. Jena, S. Chakraverty, M. Yavuz, and T. Abdeljawad, "A new modeling and existence-uniqueness analysis for Babesiosis disease of fractional order," Modern Physics Letters B, vol. 35, 2021.

[31] S. T. M. Thabet, M. S. Abdo, and K. Shah, "Theoretical and numerical analysis for transmission dynamics of COVID-19 mathematical model involving Caputo-Fabrizio derivative," Advances in Difference Equations, vol. 2021, no. 1, p. 184, 2021.

[32] K. Shah, R. U. Din, W. Deebani, P. Kumam, and Z. Shah, "On nonlinear classical and fractional order dynamical system addressing COVID-19," Results in Physics, vol. 24, p. 104069, 2021.

[33] S. K. Ntouyas and J. Tariboon, "Fractional boundary value problems with multiple orders of fractional derivatives and integrals," The Electronic Journal of Differential Equations, vol. 2017, no. 100, pp. 1-18, 2017.

[34] L. Xu, Q. Dong, and G. Li, "Existence and Hyers-Ulam stability for three-point boundary value problems with Riemann-Liouville fractional derivatives and integrals," Advances in Difference Equations, vol. 2018, no. 1, p. 458, 2018.

[35] Y. Zhou, Basic Theory of Fractional Differential Equations, World Scientific Publishing Company, London, UK, 2014.

[36] M. A. Krasnoselskii, "Two remarks on the method of successive approximations," Uspekhi Matematicheskkh Nauk, vol. 10, pp. 123-127, 1955.

[37] A. Granas and J. Dugundji, Fixed Point Theory, SpringerVerlag, New York, NY, USA, 2004. 